\title{
The electric vehicle routing problem with backhauls
}

\author{
Mauricio Granada-Echeverri ${ }^{a^{*}}$, Luis Carlos Cubides ${ }^{a}$, Jésus Orlando Bustamante ${ }^{b}$
}

${ }^{a}$ Electrical Engineering Program, Universidad Tecnológica de Pereira, Risaralda, Colombia

${ }^{b}$ Information and Communication Technologies Management Audifarma SA, Pereira, Risaralda, Colombia

\begin{tabular}{l}
\hline C H R O N I C L E \\
\hline Article history: \\
Received May 142019 \\
Received in Revised Format \\
May 142019 \\
Accepted June 52019 \\
Available online \\
June 5 2019 \\
\hline Keywords: \\
Electric vehicle routing problem \\
Mixed integer linear programming \\
Backhaul \\
Linehaul \\
VRPB
\end{tabular}
A B S T R A C T

\begin{abstract}
In the classical vehicle routing problem with backhauls (VRPB) the customers are divided into two sets; the linehaul and backhaul customers, so that the distribution and collection services of goods are separated into different routes. This is justified by the need to avoid the reorganization of the loads inside the vehicles, to reduce the return of the vehicles with empty load and to give greater priority to the customers of the linehaul. Many logistics companies have special responsibility to make their operations greener, and electric vehicles (EVs) can be an efficient solution. Thus, when the fleet consists of electric vehicles (EVs), the driving range is limited due to their battery capacities and, therefore, it is necessary to visit recharging stations along their route. In this paper the electric vehicle routing problem with backhauls (EVRPB) is introduced and formulated as a mixed integer linear programming model. This formulation is based on the generalization of the open vehicle routing problem considering a set of new constraints focussed on maintaining the arborescence condition of the linehaul and backhaul paths. Different charging points for the EVs are considered in order to recharge the battery at the end of the linehaul route or during the course of the backhaul route. Finally, a heuristic initialization methodology is proposed, in which an auxiliary graph is used for the efficient coding of feasible solutions to the problem. The operation and effectiveness of the proposed formulation is tested on two VRPB instance datasets of literature which have been adapted to the EVRPB.
\end{abstract}

() 2020 by the authors; licensee Growing Science, Canada

\section{Introduction}

The vehicle routing problem with backhauls (VRPB) can be defined as the problem of determining a set of vehicle routes to visit all customer vertices, which are divided into two subsets. The first subset contains the vertices of the linehaul customers, each requiring a given quantity of products to be delivered. The second subset contains the backhaul customers, where a given quantity of inbound products must be picked up and transported to the depot. The VRPB objective is to determine a set of vehicle routes to visit all customers in order to satisfy the demand of goods. In such a case, the vehicles must attend first the customers with delivery requirements before the customers with collection requirements. This customer division is extremely frequent in practical situations in which it is required to avoid the permanent reorganization of the goods transported and the linehaul customers have a higher priority.

* Corresponding author

E-mail: magra@utp.edu.co (M. Granada-Echeverri)

2020 Growing Science Ltd.

doi: $10.5267 /$ j.ijiec.2019.6.001 
Traditional subtour-elimination constraints fit perfectly into VRPs modeled with a single set of vertices, where the evaluation of the flow conservation and degree constraints can be made in a general way on all vertices. In VRPB, the precedence constraint stipulates that in each circuit the linehaul vertices precede the backhaul vertices. This leads to consider some special cases, such as the vertices at the end of a lineahaul route, the vertices at the start of a backhaul route and the routes with lineahaul customers only. Because of the above, and considering that the problem is known to be NP-hard in the strong sense, most of the existing literature about VRPB is related to heuristic and metaheuristic methodologies (Ropke $\&$ Pisinger, 2006). Few jobs concerning the exact approaches have been proposed and all of them focused on the inclusion of bounding techniques or set-partitioning models (Toth \& Vigo, 2014).

Thus, we have approached the problem from another point of view; considering a representation of each part of the VRPB based on a generalization of the open vehicle routing problem (OVRP). The OVRP was first proposed in the early 1980s (Schrage, 1981; Bodin et al., 1983) when there were cases where a delivery company did not own a vehicle fleet or its fleet was inadequate for fully satisfying the customers' demand. Therefore, the contractors who were not employees of the delivery company used their own vehicles for the deliveries. In these cases, the vehicles were not required to return to the central depot after their deliveries because the company was only concerned with reaching the last customer. Thus, the goal of the OVRP is to design a set of Hamiltonian paths to satisfying customers' demand.

In the VRPB, the linehaul routes constitute a subproblem that has an arborescent configuration formed by a minimum spanning tree; starting from the depot, spanning all the linehaul customers, and ending up at a linehaul customer (Toro et al., 2017a, 2017b; Lourenco et al., 2002). Note that a spanning tree becomes a subgraph formed only by Hamiltonian paths if each customer node has a degree less than or equal to two. Similarly, the backhaul routes also have an arborescent configuration, entering the depot and spanning all the backhaul customers. Thus, the VRPB structure can be seen as OVRPs of linehaul and backhaul routes connected by tie-arcs.

With the progress of technology and ecological concerns, electricity has become a solid option for fuel replacement. Electric vehicles (EVs) are considered an alternative to implement in the transport sector, some advantages of using EVs are: i) the decrease of greenhouse gas release, ii) the reduction in the dependence of fossil fuels and iii) the little noise generated. However, the EVs still have to overcome some problems associated with the battery's autonomy, since the technology still needs to grow, and with the infrastructure of the charging stations, which are not yet installed massively. Thus, integrated planning of routes and charging stations is a problem that has been gaining great importance in the transport industry in the last years: Ge et al. (2011); Dharmakeerthi et al. (2012); Liu et al. (2013); Wang et al. (2013); Paz et al. (2018); Arias et al. (2017).

Several companies have already deployed electric delivery truck fleets. Generally, the fleet is made up of the kind of medium-duty commercial delivery trucks often used to deliver supplies to customers within one locality. It is a job particularly well-suited to electric trucks for several reasons: daily routes are often exactly the same, meaning range needs are fixed and predictable, and the vehicles always return to a charging station at night, making recharging easier. Additionally, because its parcel delivery trucks are not in operation overnight, the companies do not rely on public charging infrastructure (Electrification Coalition, 2012). Some studies analyze the actual use of EVs in commercial fleets from the point of view of the maximum necessary range of autonomy of the battery to cover most of the trips. In Pfriem and Gauterin (2013), the data suggests that about $90 \%$ of the mobile days could be covered with an EV range of $60 \mathrm{~km}$ and night recharge. They show a daily mobility far below their maximum range with long parking hours at night. Likewise there is no need for fast-charging.

Thus, a topic of great interest for transport companies with EV fleets is the planning of routes considering: i) an electric truck fleet, ii) a higher priority in the linehaul customers, iii) a slow recharge at a charging 
point (CP) which is owned by the company (where the driver can rest or perform other activities) and iv) the return of the vehicle to the central depot serving backhaul customers.

This paper proposes a VRPB with a fleet consisting exclusively of EVs, where the customers with delivery requirements should not be affected by the recharge time of the battery in the charging stations, because these have a higher priority. The EVs must be recharged at the end of the linehaul route or during the course of backhaul route. Additionally it is important that the recharge takes place after the EV has covered a predetermined minimum distance, in order to take greater advantage of the initial charge of the battery. We have named this problem as electric vehicle routing problem with backhaul (EVRPB) and it is formulated as a mixed integer linear programming (MILP). The main characteristic of the proposed model is that the topological configuration of the solution is taken into account to efficiently eliminate the possibility of generating solutions formed by subtours. In order to solve the cases of greater complexity and size, a heuristic initialization methodology is proposed in which an auxiliary graph is used for the efficient decoding of feasible solutions to the problem given by a permutation.

The rest of the paper is organized as follows. In Section 2 we describe the literature review, presenting the contributions found. In Section 3 we present the problem formulation, presenting the nomenclature for the variables and parameters used in the mathematical model, also, we describe the model conditions and introduce the new mixed integer linear programming (MILP) formulation. In section 4, the initialization methodology, based on ILS metaheuristic and an auxiliary graph is presented. In Section 5 we present a computational study performed on 40 new proposed instances for the EVRPB. Finally, the conclusions are presented.

\section{Literature review}

Because the VRPB is NP-hard in the strong sense (Toth and Vigo, 2014), a lot of heuristic processes are appropriate for its solution and, therefore, most existing literature on the VRPB is related to heuristic and metaheuristic methodologies with high quality results. Two comprehensive reviews of metaheuristic techniques for VRPB are found in Ropke and Pisinger (2006). Two literature reviews cover the main works about VRPB: the first, presented by Toth and Vigo (2002), presents the existing work up to 2002 and the second, by Irnich et al. (2014) focuses on complementing the review up to 2013.

Goetschalckx and Jacobs-Blecha (1989) developed an integer programming formulation for the VRPB by extending the formulation of Fisher et al. (1986) to include pickup points. They develop a heuristic solution algorithm for this problem which, in turn, is broken into three subproblems. The first two subproblems correspond to the clustering decisions for the delivery customers and the pickup customers, which are independent generalized assignament problems. The third subproblem consists of solving $\mathrm{K}$ independent Traveling Salesman Problem (TSP) conformed by delivery and pickup customers, considering the precedence constraints, which impose a dependency relationship on all the model components.

The first exact method is reported by Toth and Vigo (1997), in which an effective Lagrangian bound is introduced that extends the methods previously proposed for the capacitated VRP (CVRP). The resulting Branch-and-Bound algorithm is able to solve problems with up to 70 customers in total. The second exact method is proposed by Mingozzi et al. (1999), in which a set-partitioning-based approach is presented and the resulting mixed integer linear programming (MIP) is solved through a complex procedure. The results show that the approach is capable of solving undirected problems with up to 70 customers. Toth and Vigo state that no exact approaches have been proposed for VRPB during the last decade (Toth and Vigo, 2014). In our review, we have reached the same conclusion and new proposals for unified exact models of VRPB were not found, since the only two existing proposals are used to derive the relaxations on which the exact approaches are based (Toth and Vigo, 1997). 
Ropke and Pisinger (2006) proposed a unified model that is capable of handling most of the variants of the VRPB, they use different metaheuristic techniques for VRPB. Chávez et al. (2018), present a Tabu search metaheuristic to solve the routing problem, they divided it into sub-routes, one for linehaul customers and one for backhaul customers, in order to obtain a global solution for the minimum cost. Chávez et al. (2016), present a multiobjective ant colony algorithm for the Multi-Depot Vehicle Routing Problem with Backhauls (MDVRPB) where three objectives are minimized:

i) the traveled distance, ii) the traveling times and iii) the total consumption of energy.

Other two problems in the literature commonly handled by exact methods, where the backhaul load is considered, are: i) the mixed vehicle routing problem with backhauls (MVRPB) and ii) simultaneous pickups and deliveries. In the first, deliveries after pickups are allowed where the linehaul and backhaul customers are mixed along the routes. In the second, the customers may simultaneously receive and send goods. Although the differences between these two problems and the VRPB appear to be subtle, they are very different; direct comparisons between the problems serving pickups and deliveries in a mixed order or simultaneously with problems where the delivery is first and the pickup second should not be performed, since they are addressing different requirements. The VRPB is a problem with a special structure of the routes that consist of two distinct parts; a delivery and a pickup segment. A complete review of these two types of problems can be found in (Ropke \& Pisinger, 2006; Wade \& Salhi, 2003; Parragh et al., 2008).

A recent survey paper with interesting conclusions and research perspectives on the VRPB, including models, exact and heuristic algorithms, variants, industrial applications and case studies, are identified in (Koç \& Laporte, 2017). In this review, the authors highlight the importance of using matheuristic algorithms that allow the interoperation of metaheuristic and mathematical programming techniques. Additionally, they identify the need for new studies focused on developing effective and powerful exact methods to solve all available standard VRPB instances to optimality. The authors also conclude that no electric vehicle version has yet been studied for the VRPB. The OVRP has recently received increasing attention in the literature and has focused mainly on the development of heuristic methods to find good quality solutions quickly. Regarding the exact methods, a branch-and-cut algorithm for the open version of the CVRP, addressing the capacitated problem with no distance constraints is proposed by Letchford et al. (2007). Pessoa et al. (2008) present several branch-cut-and-price algorithms on a number of vehicle routing problem variants, among which is the capacitated OVRP, which is addressed by setting the cost of all arcs that have the depot as the endpoint to zero. Salari et al. (2010) proposed a heuristic improvement procedure for the OVRP based on integer linear programming techniques to improve a feasible solution of a combinatorial optimization problem. Alinaghian et al. (2016) proposed a mathematical model in which open paths are used into the problem of cross-docks. To model the open path, a dummy node is defined, whose distance to other nodes is considered zero, and from which the route starts. A comprehensive literature review on the OVRP is presented in (Li et al., 2007; Toro et al., $2017 b, a)$.

In relation to EVs, in the context of the VRP, Yang and Sun (2015) present an electric vehicle battery swap station location routing problem (BSS-EV-LRP), which aims to determine the location strategy of battery swap stations (BSSs) and the routing plan of a fleet of electric vehicles (EVs) simultaneously under battery driving range limitations, a four-phase formulated heuristic technique, called SIGALNS, is proposed to solve the problem. Goeke and Schneider (2015) propose the Electric Vehicle Routing Problem with Time Windows and Mixed Fleet (E-VRPTWMF) to optimize the routing of a mixed fleet of electric commercial vehicles (ECVs) which assume energy consumption to be a linear function of the distance traveled and the recharging times at stations by time windows. Arias et al. (2017) present a probabilistic approach for the optimal charging of electric vehicles (EVs) in distribution systems, where the costs of both demand and energy losses in the system are minimised, subjected to a set of constraints that consider EVs smart charging characteristics and operative aspects of the electric network. 
Finally, regarding the decoding of a permutation in the context of the VRP, Ochi et al. (1998) adopt a representation where depots are used as trip delimiters. A more straightforward way is to use a sequence of customers without trip delimiters, as has been done for the CVRP by Liu et al. (2008). When the vehicles are homogeneous, Prins (2004) developed a polynomial time procedure for deliveringa single product, using the shortest path problem on an auxiliary acyclic graph. In (Vidal et al., 2012; Cattaruzza et al., 2014), the authors present a procedure based on an adaptation of the procedure proposed by Prins, which also works on an auxiliary graph. Due to the existence of precedence constraints, the limited battery capacity and the different types of existing vertices on the EVRPB, the procedure proposed in Prins (2004) cannot be directly used and it is modified as explained in Section 4.2.

\section{Proposed model for the EVRPB}

\subsection{Problem formulation}

Fig. 1 shows the optimal solution of an VRPB with 25 customers; in which the first 20 customers (numbered from 1 to 20 and represented by circles) are linehaul customers and the other 5 (numbered from 21 to 25 and represented by squares) are backhaul customers. The depot is the vertex 0 and the dotted lines indicate the connecting arcs that connect the linehaul with the backhaul customers. For this instance, the capacity of all vehicles is equal to $Q=1550$. The minimum number of vehicles needed to serve all the linehaul and backhaul customers is $\mathrm{K}_{\mathrm{L}}=8$ and $\mathrm{K}_{\mathrm{B}}=2$, respectively. These values can be obtained by solving the bin packing problem instances associated with the corresponding customer subset, which calls for the determination of the minimum number of bins, each with capacity Q, needed to serve all customers (Toth and Vigo, 2002). To ensure feasibility, we assume that the number of vehicles needed $\mathrm{K} \mathrm{V}$ must be greater than or equal to the maximum value between $\mathrm{K}_{\mathrm{L}}$ and $\mathrm{K}_{\mathrm{B}}$. The demand (delivered or collected) of each customer is shown in the figure with the notation $(\cdot)$. Thus, the basic version of the VRPB must satisfy the following conditions:

- Each vertex must be visited exactly once by a single route. That is, each vertex has degree 2 .

- Each route starts and finishes at the depot.

- Each customer must be fully attended when visited.

- All customers are serviced from a single depot.

- The vehicle capacity should never be exceeded in both the linehaul and backhaul route and all vehicles should have the same capacity.

- In each circuit the linehaul vertices precede the backhaul vertices (precedence constraint), if any. That is:

- A circuit of only backhaul customers is not allowed.

- The last customer of a linehaul route is always connected to the depot or to a backhaul customer (BC) who is starting a backhaul route.

- The last $\mathrm{BC}$ of a backhaul route is always connected to the depot. 


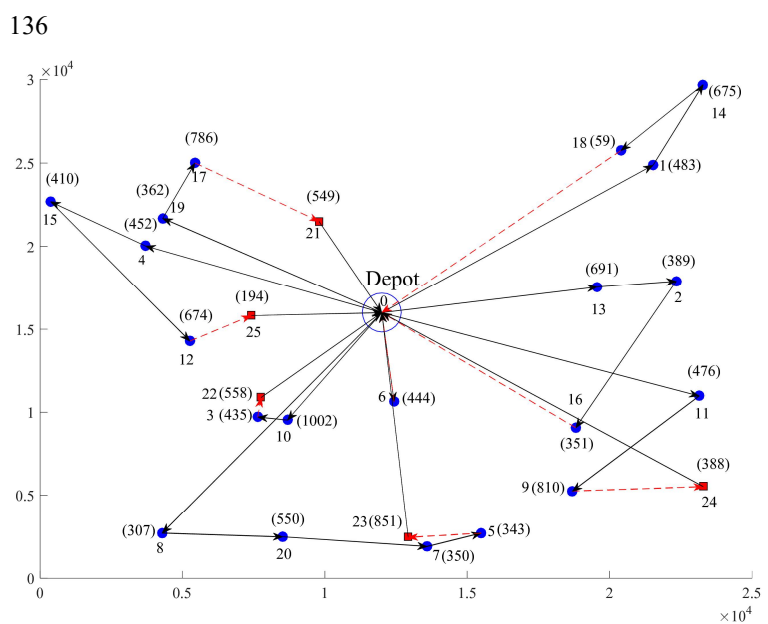

Fig. 1. Vehicle routing problem with backhaul (VRPB)

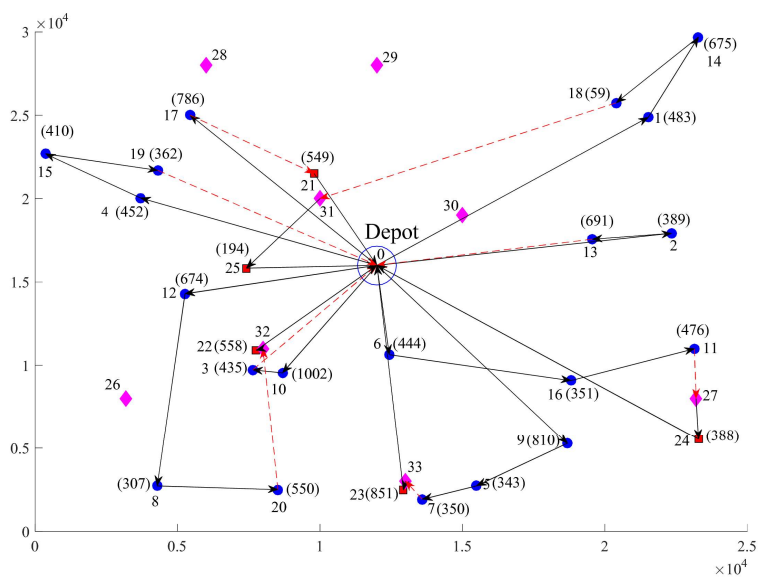

Fig. 2. Electric vehicle routing problem with backhaul (EVRPB)

In the EVRPB, when the electric vehicle ends the linehaul route, the driver can follow several alternatives: i) start the backhaul route, ii) return directly to the depot, or iii) rest in the charging point and recharge the battery in slow mode until the next day. Figure 2 shows the optimal solution of an EVRPB where charging points are represented by diamonds, numbered from 26 to 33 . Thus, when the charging points and battery life of the EV are considered, the EVRPB must, aditionally, satisfy the following conditions:

- Each charging point must be visited by one or more routes, or never be visited.

- The EVs are fully charged in the depot and in the charging points.

- The charging points are visited, only if it is necessary, at the end of the linehaul customers or during the course of the backhaul route.

- The charging stations are already built and their demand is equivalent to zero.

\subsection{Nomenclature}

The nomenclature for the sets, variables and parameters of the proposed model for the EVRPB is summarized next.

Sets:

$L$

$B$

$K$

$L_{o}$

$B_{o}$

$C_{u}$

V

Parameters:

$M_{i j}$

$C_{i j}$

$D_{j}$

$K_{V}$

$Q$

$E^{\max }$

Variables:

$s_{i j}$

$\xi_{i j}$
Set of linehaul customers. $L=\{1, \ldots, n\}$.

Set of backhaul customers. $B=\{n+1, \ldots, n+m\}$.

Set of charging points. $K=\{n+m+1, \ldots, n+m+k\}$.

Set of linehaul customers and the depot $L_{o}=\{0\} \cup L$. Vertex 0 corresponds to the depot.

Set of backhaul customers, depot and charging points. $B_{o}=\{0\} \cup B \cup K$.

Set of linehaul and backhaul customers, including the charging points. $C_{u}=L \cup B \cup K$.

Set of Nodes $V=\{0\} \cup C_{u}$.

Distance between nodes $i$ and $j$.

Cost of traveling between nodes $i$ and $j$.

Nonnegative quantity of products to be delivered or collected (demand) of the customers $j \in C_{u}$.

Number of available vehicles (given in advance).

Capacity in goods of the vehicles.

Electric capacity of the vehicles (identical vehicles).

Binary variable for the use of the path between nodes $i, j \in V$.

Binary variable for the use of the path between nodes $i \in L$ and $j \in B_{o}$ 
$l_{i j} \quad$ Continuous variable indicating the amount of goods transported between nodes $i$ and $j$.

$p_{i j}$ Distance accumulated by the electric vehicle from the depot to the arc $(\mathrm{i}, \mathrm{j})$ nodes $i$ and $j$.

$p_{j}^{L}$ Auxiliary variable that indicates the distance between the linehaul customers $j\left(\mathrm{LC}_{\mathrm{j}}\right)$ and the depot. For a $\mathrm{BC}$ or a $\mathrm{CP}$, this distance is denoted by the variables $p_{j}^{B}$ and $p_{j}^{K}$, respectively.

\subsection{Proposed Model for the EVRPB}

The EVRPB can be defined as the following graph theoretic problem. Let $G=(V, A)$ be a complete and directed graph, where $V=0 \cup C_{u}$ is the vertex set and $A$ is the arc set. The vertex 0 denote the depot and vertex set $C_{u}$ represents the feasible points that the EV can visit, once it leaves the depot. These feasible points are conformed by: the set of $n$ linehaul customers (LCs), defined as $L=\{1,2, \ldots, n\}$, the set of $m$ backhaul customers (BCs), defined as $B=\{n+1, \ldots, n+m\}$ and the set of $k$ charging points (CPs), defined as $K=\{n+m+1, \ldots, n+m+k\}$. Thus, $C_{u}=L \cup B \cup K$ where each vertex $j \in C_{u}$ is associated with a known nonnegative demand of goods $D_{j}$ to be delivered or collected, considering that if $j \in K$ then $D_{j}=0$. The depot has an unlimited fleet of identical vehicles with the same positive load capacity, denoted as $Q$, and the same electric capacity, denoted as $E^{\max }$. The number $K_{V}$ of vehicles for use is given in advance.

This mathematical formulation corresponds to a commodity flow model that uses two binary decision variables: $s_{i j}$ that takes value 1 if $\operatorname{arc}(i, j) \in A$ belongs to the optimal solution and $\xi_{i j}$ that takes value 1 if the tie-arc between nodes $i \in L$ and $j \in B \cup 0$ is used. The tie-arcs connect the linehaul routes with the backhaul routes. The nonnegative flow variable $l_{i j}$ is associated with the flow of goods transported by a vehicle through the arc $(i, j) \in A . p_{i j}$ is a continuous variable indicating the EVs state of charge in distance units between nodes $i$ and $j . p_{j}^{L}$ is a continuous auxiliary variable that represents the distance between the linehaul node $j$ and the depot.

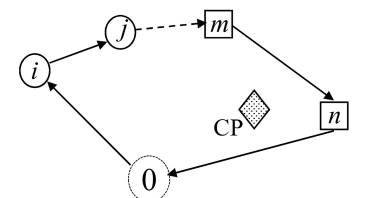

(a) Without charging point

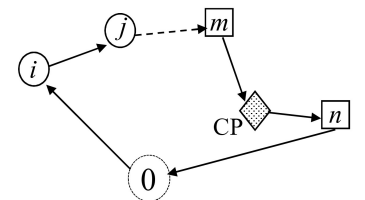

(b) $\mathrm{CP}$ in the backhaul route

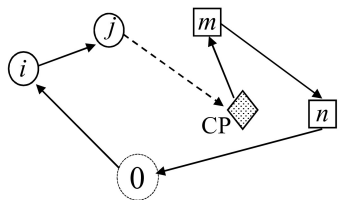

(c) $\mathrm{CP}$ at the end of the linehaul route

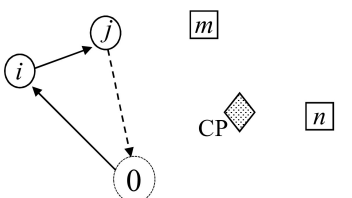

(d) Only linehaul customers

Fig. 3. Types of routes for the EVRPB

The commodity flow model (1)-(34) is an integer linear programming formulation of the EVRPB proposed. Figure 3, described the types of routes that can be found in the solution of EVRPB, where circles represent LCs, squares represent BCs and diamonds represent CPs. The EVRPB objetive is to minimize the total cost of routes needed to visit all customers or charging points.

$$
\min =\sum_{i \in V} C_{i j} \times s_{i j}+\sum_{\substack{i \in L \\ j \in B_{o}}} C_{i j} \times \xi_{i j}
$$

subject to

$$
\begin{array}{ll}
\sum_{i \in L_{o}} s_{i j}=|L| & \\
\sum_{i \in L}^{j \in L_{o}} l_{i j}-\sum_{k \in L} l_{j k}=D_{j} & \forall j \in L \\
\sum_{i \in L_{o}} s_{i j}=1 & \forall j \in L
\end{array}
$$




$$
\begin{aligned}
& 138 \\
& \sum_{k \in L_{o}} s_{j k}+\sum_{k \in B_{o}} \xi_{j k}=\sum_{i \in L_{o}} s_{i j} \quad \forall j \in L \\
& l_{i j} \leq Q \times s_{i j} \quad \forall i \in L_{o}, \quad \forall j \in L \\
& \sum_{j \in L_{o}} s_{0 j} \geq \frac{\sum_{j \in L} D_{j}}{Q} \\
& \sum_{i \in L} s_{0 j}=K_{V} \\
& p_{j}^{L}=\sum_{i \in L_{o}} M_{i j} * s_{i j} \quad \forall j \in L \\
& \sum_{i \in L_{o}} p_{i j}-\sum_{k \in V} p_{j k}=p_{j}^{L} \quad \forall j \in L \\
& p_{i j} \leq E^{\max } \times s_{i j} \quad \forall i \in L_{o}, \quad \forall j \in L \\
& p_{i j} \leq E^{\max } \times \xi_{i j} \quad \forall i \in L, \quad \forall j \in B \\
& p_{0 j}=E^{\max } \times s_{0 j} \quad \forall j \in L \\
& p_{j 0} \geq D_{j 0} \times \xi_{j 0} \quad \forall j \in L \\
& \sum_{i \in B} s_{i j}=|B| \\
& \sum_{i \in B}^{j \in B_{o}} l_{i j}-\sum_{k \in B_{o}} l_{j k}=-D_{j} \quad \forall j \in B \\
& \begin{array}{l}
\sum_{j \in B_{o}} s_{i j}=1 \forall i \in B \\
\sum_{k \in B} s_{k i}+\sum_{j \in L} \xi_{j i}+\sum_{m \in K} s_{m i}=\sum_{j \in B_{o}} s_{i j} \quad \forall i \in B
\end{array} \\
& l_{i j} \leq Q \times s_{i j} \quad \forall i \in B_{o}, \quad \forall j \in B_{o} \\
& \sum_{i \in B} s_{i 0} \geq \frac{\sum_{j \in B} D_{j}}{Q} \\
& \sum_{i \in B} s_{i 0}+\sum_{i \in L} \xi_{i 0}=\sum_{j \in L} s_{0 j} \\
& p_{j}^{B}=\sum_{i \in B} M_{i j} s_{i j}+\sum_{i \in L} M_{i j} \xi_{i j}+\sum_{i \in K} M_{i j} s_{i j} \quad \forall j \in B \\
& \sum_{i \in C_{u}} p_{i j}-\sum_{k \in B_{o}} p_{j k}=p_{j}^{B} \quad \forall j \in B \\
& \begin{array}{lll}
p_{i j} \leq E^{\text {max }} \times s_{i j} & \forall i \in B, & \forall j \in B_{o} \\
p_{j 0} \geq D_{j 0} \times s_{j 0} & \forall j \in B &
\end{array} \\
& \sum_{k \in B} s_{k i}+\sum_{j \in L} \xi_{j i}=\sum_{j \in B} s_{i j} \quad \forall i \in K \\
& \sum_{i \in B} l_{i j}-\sum_{k \in B_{o}} l_{j k}=0 \quad \forall j \in K \\
& p_{j}^{K}=\sum_{i \in B} M_{i j} \times s_{i j}+\sum_{i \in L} M_{i j} \times \xi_{i j} \quad \forall j \in K \\
& \sum_{i \in C_{u}} p_{i j}-\sum_{k \in K_{o}} p_{j k}=p_{j}^{K} \quad \forall j \in K \\
& \begin{array}{lll}
p_{i j} \leq E^{\max } s_{i j} & \forall i \in K, & \forall j \in B \\
s_{i j}+s_{j i} \leq 1 & \forall i \in V, & \forall j \in V \\
\xi_{i j} \in\{0,1\} & \forall i \in L, & \forall j \in B_{0} \\
s_{i j} \in\{0,1\} & \forall i, j \in V &
\end{array}
\end{aligned}
$$




$$
l_{i j} \in R
$$

The objective function (1) minimises the operating costs and consist of 2 terms. The first corresponds to the sum of the total travelling cost of the routes used to deliver and collect the goods and to visit the charging points. The second corresponds to the use of the tie-arcs connecting the last customer of a linehaul route to the backhaul customer, to the charging point or to the depot.

The sets of constraints (2)-(8) allow modelling the OVRP for linehaul routes, where (2) and (3) impose the connectivity requirements. In the optimal solution of the OVRP, each route has an arborescent configuration formed by a minimum spanning tree; starting from the depot, spanning all the nodes, and ending at a customer. We have named this subproblem the linehaul open vehicle routing problem (LOVRP). In the vehicle routing problem context, the necessary condition for obtaining a minimum spanning tree is that the number of arcs be equal to the number of customer nodes. This necessary condition is guaranteed by the equality constraint (2), where the number of customer nodes is given by the cardinality of the set $L$. However, this constraint is necessary but not sufficient because there may be customer nodes with a degree greater than two and disconnected solutions can be obtained.

A spanning tree becomes a subgraph formed only by Hamiltonian paths if each customer node has a degree less than or equal to two. Therefore, another necessary condition is given by the sets of degree constraints (4) and (5). The indegree constraints (4) impose that exactly one arc enters each customer node and, consequently, the outdegree constraints (5) impose that exactly one arc leaves each LC, considering two situations: i) a tie-arc can only go from a LC towards a BC or towards the depot and ii) only a arc coming from a LC or from the depot can arrive at a LC. However, the addition of these degree constraints in directed graphs may not represent a spanning tree, because a disconnected graph can be obtained.

The addition of the flow balance constraint by each customer node avoids getting disconnected solutions, since an infeasible solution is obtained when the goods leaving the depot can not reach the LCs. Thus, the set of constraints reported in (3) guarantees network connectivity through the flow conservation constraint for each LC so that they are fully served when visited. Similarly, the constraints (16) and (27) guarantees network connectivity through the balance of the demand flow by each $\mathrm{BC}$ and charging point, respectively. Note that in the constraints (27) the demand for the $\mathrm{CP}$ is considered to be 0 .

The constraints (6) and (7) impose both the vehicle and depot capacity requirements, respectively. The first is an upper limit defined by the capacity of the vehicle to transport a quantity of products on any linehaul-arc, while the second is a lower limit to the number of routes out of the depot to supply linehaul customers, which is determined by the ratio between the total demand to be collected and the vehicle capacity. Constraint (8) limits the minimum number of vehicles used on linehaul routes.

Similarly to the sets of constraints (2)-(8), are established the sets of constraints (15)-(20) for modeling the OVRP for backhaul routes (BOVRP). The set of constraints (21) ensures that the number of arcs leaving the depot is equal to the number of arcs coming to depot. Comparing (21) and (8) one can see that the number of linehaul arcs leaving the depot may be different from the number of backhaul arcs arriving at the depot. This case occurs when there are tie-arcs between a linehaul route and the depot.

The sets of constraints (9)-(14) represent the limitations of EVs when crossing a route of LCs. The constraints (9) and (10) guarantee the fulfillment of the distance balance constraint on a LCs route, which is necessary for the calculation of the accumulated distance at the moment of crossing every arc $(i, j)$ of the optimal solution. Similarly, constraints (22) and (23) guarantee the fulfillment of the distance balance constraint on a BCs route, and (28) and (29) do the same for the set of vertices that are CPs.

The sets of constraints (11) and (12), ensure that when an arc between LCs or a tie-arc is crossed, respectively, the maximum capacity of the vehicles battery, in terms of distance, is not exceeded. 
Similarly, the sets of constraints (24) and (30) verify compliance with this same electrical capacity constraint when an arc between $\mathrm{BCs}$ or between a $\mathrm{CP}$ and a $\mathrm{BC}$ is crossed, respectively.

The set of equations (13) ensures that the EV leaves the depot with the battery fully charged. The return to the depot is always done through a tie-arc or an arc coming out of a backhaul node. Therefore, the constraints (14) ensure that the battery charge is sufficient to return to the depot through a tie-arc. The constraints (25) do this same verification when the EV returns to the depot through an arc that leaves a node backhaul.

The set of equations (26) imposes that the number of arcs arriving and leaving a CP is the same, considering two situations: i) that a tie-arc from an $\mathrm{LC}$ or $\mathrm{BC}$ can arrive at a $\mathrm{CP}$ and ii) that from a $\mathrm{CP}$ an arc can only be connected to a $\mathrm{BC}$. The direct return from a $\mathrm{CP}$ to the depot is not allowed since the objective is to take advantage of the total charge of the EV to make a backhaul route, and not only to return to the depot. Note that this constraints are similar to (18), which impose that exactly one arc leaves each $\mathrm{BC}$ visited. In (18) two situations are considered: i) that an arc that arrives at a $\mathrm{BC}$ can only come from another $\mathrm{BC}$, from a tie-arc that leaves an $\mathrm{LC}$ or from a $\mathrm{CP}$, and ii) that an arc from a $\mathrm{BC}$ can only be connected to another $\mathrm{BC}$ or to the depot.

Finally, the constraints (31) ensure that only one of the two variables $s_{i j}$ or $s_{j i}$ be used. Constraints (32) and (33) define all binary decision variables, and constraints (34) define the real variables. The mathematical model (1)-(34) can represent the classic VRPB, when the capacity of the battery is not considered ( $E^{\mathrm{max}}$ large enough).

\section{Proposed model for the EVRPB}

The computational results obtained on several test instances, show that some cases configure a highly restricted problem, where obtaining a feasible integer initial solution requires high computational times. This can be evidenced in Tables 1 and 2, in Section 5. Thus, the purpose of the initialization phase is to quickly find a feasible integer initial solution through an efficient heuristic algorithm in order to provide an initial upper bound to the exact algorithm used by the commercial solver.

An iterated local search (ILS) algorithm is used as an initialization methodology, whose main characteristic is to apply inter-route and intra-route movements to explore the search space generated by the solution encoding strategy. One of the key aspects in the implementation of an efficient ILS is to properly define the solution encoding strategy. In the TSP, for example, a sequence or permutation of customers turns out to be a natural and efficient representation of a feasible solution to the problem, which provides the order in which the customers (cities) should be visited and does not require additional processes of feasibility or split. In the context of the VRP, Prins (2004) presents an optimal splitting procedure (OSP) of a permutation, which in a simple and efficient way allows to obtain a solution conformed by feasible routes that leave and arrive at the depot. The algorithm consists of transforming the VRP into the shortest path problem (SPP) using an auxiliary graph constructed from the evaluation of all possible routes resulting from following the sequence given by the permutation. Therefore, a permutation generates multiple feasible solutions to the VRP, but only the best of them is chosen through the optimal solution of the SPP. Finally, the feasibility of each route in the VRP is determined by compliance with two constraints: i) vehicle capacity and ii) maximum distance traveled.

In this paper, the encoding of a solution of the EVRPB is done through a sequence of customers without trip delimiters and a modified OSP is used to decode it.

\subsection{Initial solution}

In the context of the EVRPB, a randomly generated permutation (solution) can cause a conflict with the existing precedence constraint; e.g., if the first element of the permutation corresponds to a $\mathrm{BC}$ then the 
solution is infeasible because trips leaving the depot directly to $\mathrm{BC}$ are not allowed. The same happens when the first element of the permutation corresponds to a CP.

To improve the robustness of the initial solution, a greedy strategy is proposed, where the objective is to obtain a quick and simple solution that prioritizes the following conditions: i) the trip starts with an LC, ii) from a vertex $i$, the nearest vertex $j$ must be chosen as destination, where $j \in C_{u}: j=$ $\min \left\{M_{i, j} \forall i \in C u\right\}$, and iii) the compliance of the battery autonomy constraint must be guaranteed. The general structure of the strategy is shown in Algorithm 1.

Algorithm 1. Greeddy strategy pseudo code

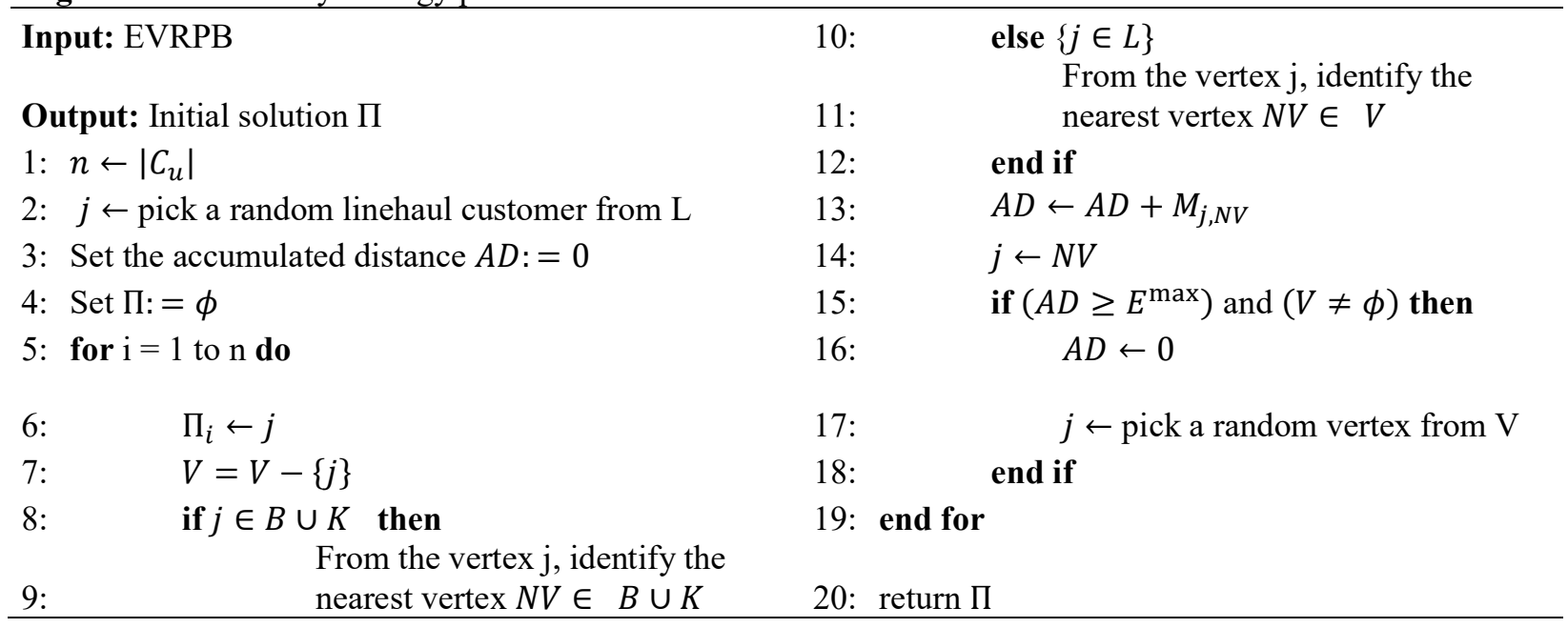

\subsection{Optimal splitting procedure with backhaul (OSPB)}

The main characteristic of the proposed OSPB is that, in addition to feasibility criteria based on the vehicle capacity, the following are also considered: i) the autonomy of the battery, ii) the precedence constraint, and iii) the minimum number of vehicles given in advance.

Given a solution $\Pi=$ permutation $\left(C_{u}\right)$, then obtaining the value of the fitness function requires the construction of an auxiliary graph $H=\left(V, A^{\prime}, T\right) . V$ is the set of vertices indexed from 0 to $\left|C_{u}\right| . A^{\prime}$ is the arc set, where each $\operatorname{arc}(i, j)$ represents a feasible trip in which the EV departs from node 0 (depot) and visits nodes $i+1, i+2, i+3, \ldots, j-1$, and $j$, consecutively. Thus, the feasible trip visiting vertices $v=\Pi_{i+1}$ to $w=\Pi_{j}$, in the order they are in $\Pi$, is denoted as $T^{v, w} \in T$. The set $A^{\prime}$ can contain a maximum number of trips $n t=\frac{1}{2}\left|C_{u}\right|\left(\left|C_{u}\right|-1\right)$. Thus, $T$ is the set of trips, where a trip $T^{v, w}$ is conformed, in turn, by $u$ vertices, which can be LCs, BCs and/or CPs, arranged in any order. The trip distance associated with $\operatorname{arc}(i, j), z_{i, j}$, is calculated according to the following equation:

$$
z_{i, j}=M_{0, v}+\sum_{k=i+1}^{i+u} M_{\Pi_{k}, \Pi_{k+1}}+M_{w, 0}
$$

However, there are different conditions that must be fulfilled so that a trip of the auxiliary graph is feasible and so that, in turn, there is a feasible solution to the SPP.

\subsubsection{Trip feasibility}

Constraints of precedence and vehicle capacity: according to the precedence constraint, a necessary condition for a trip $T^{v, w}$ to be feasible is that the first vertex corresponds to an LC. Additionally, a trip is still feasible if the first $t \leq u$ customers of the trip $T^{v, w}$ are LCs (see condition (36)) and the sum of their 
demands, $D L_{i, j}$, does not exceed the capacity of the vehicle (see constraint (38)). Another necessary conditions for trip feasibility is that after visiting the LCs only BCs or CPs can be visited (see condition (37)) and the sum of their demands, $D B_{i, j}$, does not exceed the capacity of the vehicle (see constraint (39)).

$$
\begin{aligned}
& \left\{v, \Pi_{i+2}, \Pi_{i+3}, \ldots, \Pi_{i+t}\right\} \subseteq L \\
& \left\{\Pi_{i+t+1}, \Pi_{i+t+2}, \ldots, w\right\} \subseteq\{B \cup K\} \\
& D L_{i, j}=\sum_{k=i+1}^{i+t} D_{\Pi_{k}} \leq Q \\
& D B_{i, j}=\sum_{k=i+t+1}^{i+t+u} D_{\Pi_{k}} \leq Q
\end{aligned}
$$

Battery autonomy constraint: the trip $T^{v, w}$ may contain $c$ charging points in positions $\left\{q_{1}, q_{2}, \ldots q_{c}\right\}, q_{1}<$ $q_{2}<\cdots<q_{c}$. When an EV visits a CP its battery is fully charged, which implies that the battery consumption, in terms of distance, is equivalent to the maximum distance, $E D_{i, j}$, traveled between CPs (including the depot). Therefore, the autonomy of the battery is guaranteed with the constraint (40).

$$
E D_{i, j}=\max \left\{M_{0, v}+\sum_{k=i+1}^{i+q_{1}-1} M_{\Pi_{k}, \Pi_{k+1}} ; \sum_{k=i+q_{1}}^{i+q_{2}-1} M_{\Pi_{k}, \Pi_{k+1}} ; \ldots \sum_{k=q_{c-1}}^{q_{c}-1} M_{\Pi_{k}, \Pi_{k+1}}+M_{w, 0}\right\} \leq E^{\max }
$$

\subsubsection{Construction of the auxiliary graph}

A simple example with three LCs, $L=\{1,2,3\}$, three BCs, $B=\{4,5,6\}$, and three CPs, $K=\{7,8,9\}$ is given in Fig. 4, which shows different types of solutions, in the EVRPB context, that can be obtained from the decoding of a permutation. Fig. 4a shows a grand tour, given by the permutation $\Pi=$ $\{3,2,7,6,1,8,4,9,5\}$, that starts and ends at the depot. This solution can be obtained directly from the sequence given by the permutation without the need for a split procedure. Note that this initial permutation can be obtained using Algorithm 1. It is assumed that all the arcs have a distance of $20 \mathrm{~km}$, except $M_{4,9}=50 \mathrm{~km}$. Additionally, it is considered that $M_{0, j}=20 \mathrm{~km} \forall j \in V, E^{\max }=60$ and $Q=15$. Fig. 4a shows in parentheses the quantity of products that will be delivered in the LCs or that will be collected in the BCs. Fig. 4b shows a feasible solution of EVRPB consisting of two routes. This solution is feasible because the load capacity of the vehicle is not exceeded and the autonomy of the battery is guaranteed with the charge points 7 and 8 . However, despite complying with the two previous capacity constraints, an infeasible solution can be obtained when there is a trip from the depot to a BC, as shown in Fig. 4c. The step-by-step construction of the auxiliary graph is shown in Fig. 5.

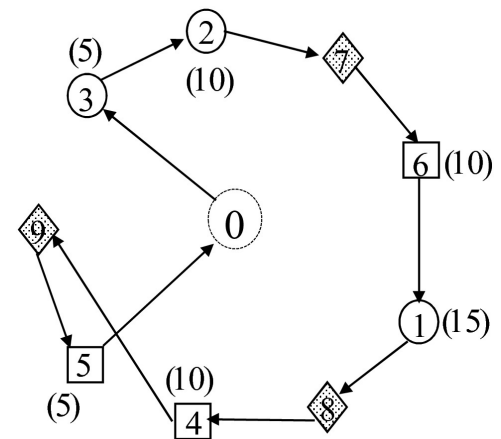

(a) Grand tour

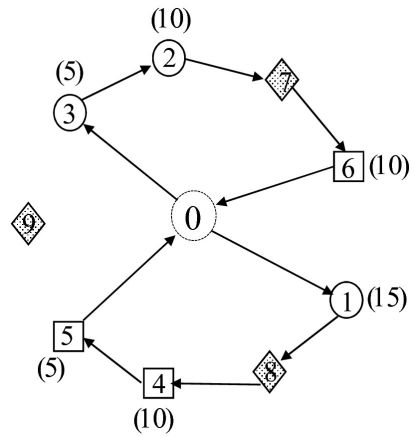

(b) Feasible solution

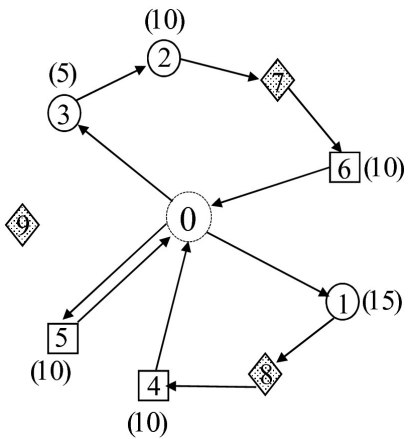

(c) Infeasible solution

Fig. 4. Types of solution 


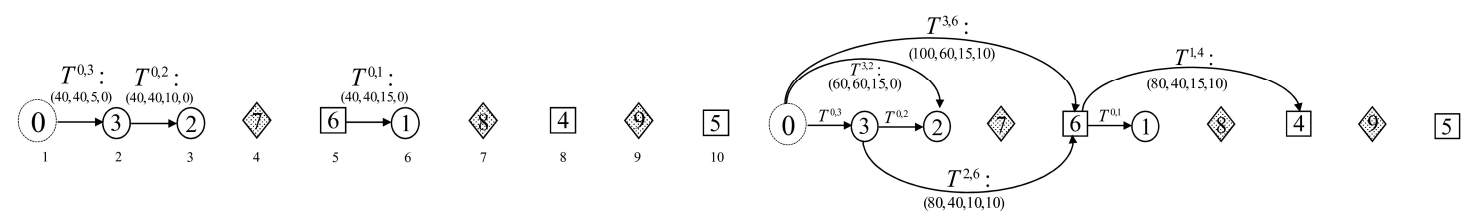

(a) Step One: express delivery to a LC

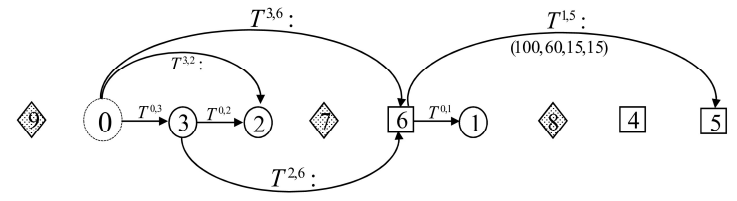

(c) Step three: infeasibility reduction by shifting a CP (b) Step Two: feasible trips from the permutation

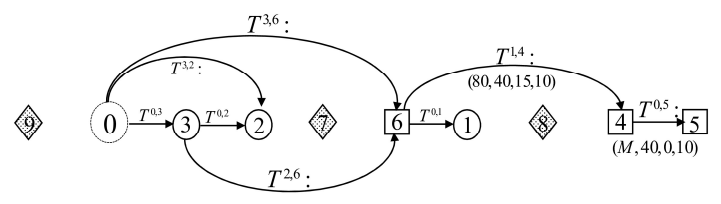

(d) Step four: infeasibility reduction by pickup express to an $\mathrm{BC}$

Fig. 5. Construction of the auxiliary graph

Initially, in step one shown in Figure 5a, all feasible trips that leave the depot, go to a LC and return to the depot are considered and stored in $A^{\prime}$. For example, the trip $T^{0,1}$ of the auxiliary graph corresponds to an EV that leaves the depot, travels $20 \mathrm{~km}$ to the LC, delivers 15 units of product and travels another $20 \mathrm{~km}$ back to the depot. In this step, the depot is the origin vertex of the SPP and corresponds to the vertex $\mathrm{i}+1$ in the auxiliary graph. Thus, the trip is characterized by a total distance of $40 \mathrm{~km}$, a battery consumption of $40 \mathrm{~km}, 15$ units delivered and 0 collected $\left(T^{0,1}:(40,40,15,0)\right)$. This trip is feasible in terms of: battery life span compared to the distance traveled (see (40)), vehicle capacity (see (39) and (38)) and precedence constraint (see (36) and (37)).

Then, in step two (see Figure 5b), all feasible trips are obtained, following the sequence given by the solution $\Pi$. For example, the trip $T^{3,2}$ corresponds to a feasible trip consisting exclusively of LCs; the trip $T^{3,7}$ is not considered because a return to the depot from a CP is not allowed; the trip $T^{3,6}$ (see Figure $4 \mathrm{~b}$ ) corresponds to a feasible trip with a path $z^{3,6}=100$, a maximum battery consumption $E D=$ $\max \{60,40\}=60 \leq E^{\max }$, and 15 units delivered and 10 collected $\left(T^{3,6}:(100,60,15,10)\right)$. Note that a trip leaving the depot to a vertex $j \in B \cup K$ is infeasible since it is not allowed to go directly from the depot to a $\mathrm{BC}$ or a $\mathrm{CP}$. All feasible trips found in this step are added to $A^{\prime}$.

\subsubsection{Feasibility of the $S P P$}

Of all the possible trips of a $\Pi$ solution, only a percentage is feasible and they make up the arc set $A^{\prime}$ of the auxiliary graph. However, in the EVRPB, obtaining a feasible solution from the SPP may not be possible since, eventually, the auxiliary graph may be disconnected. Note that, in Figure 5b, the trip $T^{1,5}$ is infeasible by battery autonomy, which produces an infeasible graph for the SPP, since there is not at least one path between the depot and the last vertex of the permutation. Therefore, the next two steps consist of a process of reduction of infeasibility, which are applied sequentially until obtaining a feasible auxiliary graph for the SPP.

Step three: if there is a CP in the position $k$ of the permutation that does not belong to a trip, in concordance with the condition (41), then this CP is shifted to the first position of the permutation.

$$
\Pi_{k} \in K: \sum_{\forall i \in V} A_{i, \Pi_{k}}=0
$$

Thus, the original permutation is modified and denoted as $\Pi^{\text {new }}=\{9,3,2,7,6,1,8,4,5\}$.

It is important to note that this step, implicitly, corresponds to a particular case of a well-known neighborhood structures so-called INSERT, which consists of removing the customer at the ith position from the permutation and then inserting it into the $j t h$ position, $i \neq j$. This case is illustrated in Figure 
5c. Note that the depot is still the origin vertex of the SPP and becomes the vertex $i+2$ in the auxiliary graph after the $\mathrm{CP}$ is shifted.

More generally, the EV departs from node 0 and visits nodes $i+$ Origin, $i+$ Origin $+1, i+$ Origin + $2, \ldots, j-1$, and $j$, consecutively, considering that Origin $=0$ for the initial permutation $\Pi$ and Origin $=$ Origin +1 each time a CP is shifted. Therefore, $A^{\prime}$ contain one $\operatorname{arc}(i, j), i<j$, if a trip from $v=$ $\Pi_{i+\text { origin }+1}$ to $w=\Pi_{j}$ is feasible.

Step four: if there is still a vertex $b$ of the permutation that does not belong to a trip, in concordance with the condition (42), then, exclusively for this BC, an express pickup with a very high distance traveled value is allowed (big M), as shown in Figures $4 \mathrm{c}$ and $5 \mathrm{~d}$ with the trip $T^{0,5}$. The value of $\mathrm{M}$ is defined as the total distance traveled by the grand tour.

$$
\Pi_{b} \in B: \sum_{\forall i \in V} A_{i, \Pi_{b}}=0
$$

\subsubsection{Integrated algorithm for the optimal split with backhaul}

The integration of all the steps, described above, allows building a connected auxiliary graph enabled to generate a feasible solution to the SPP. However, this solution could eventually be unfeasible for the EVRPB due to the need of creating a highly penalized arc in the auxiliary graph, corresponding to a direct trip from the depot to a BC (express pickup). The general structure of the OSPB strategy is shown in Algorithm 2. A binary variable $\mathrm{F}=0$ is used to indicate that the SPP solution is infeasible for the EVRPB (see line 13). When this happens, it is necessary to implement an infeasibility improvement process, for which, we propose a simple iterated local search technique.

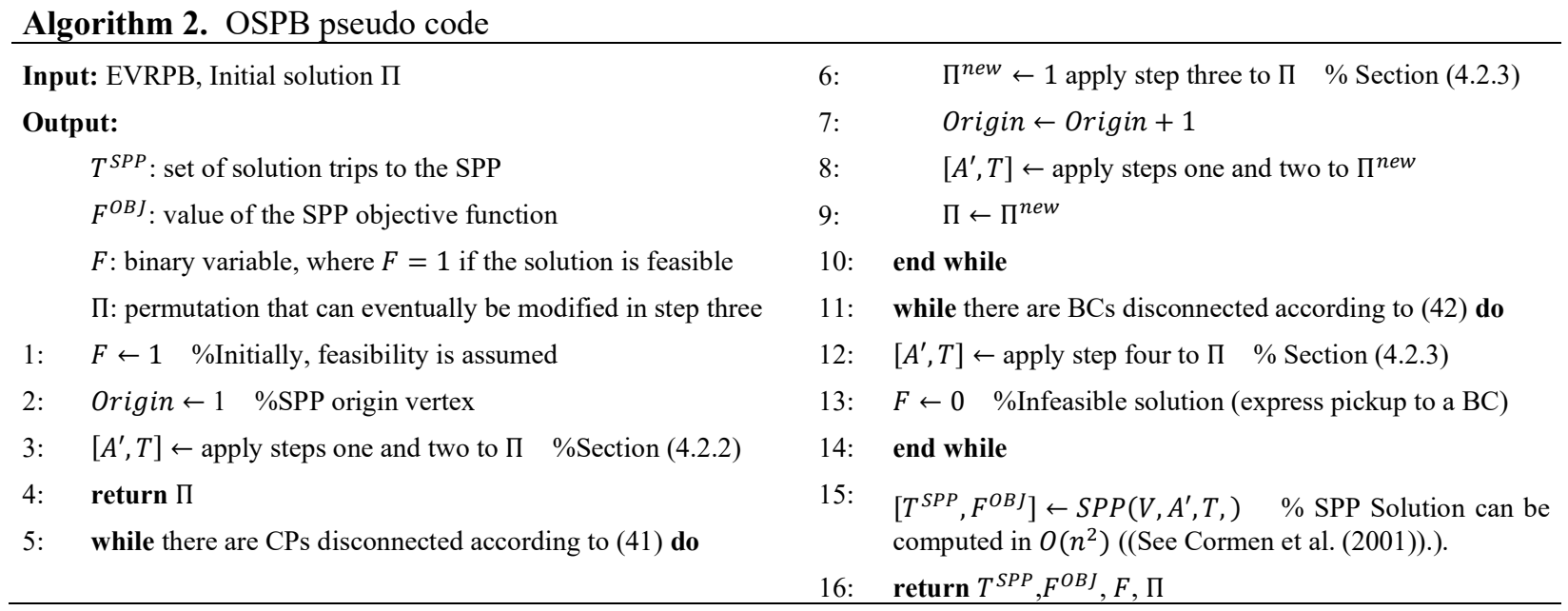

\subsection{Iterated local search}

The OSPB proposed makes it possible for the EVRPB to be coded through a permutation and, therefore, many heuristic and metaheuristic strategies can be implemented easily to obtain efficient solutions. However, our objective is to present a simple technique based on local search that allows to obtain quickly a feasible integer solution of the EVRPB, that can be used as the upper limit in the solution of the larger and more complex instances when they are resolved through the proposed MIP model.

The iterated local search technique (ILS) is a method that uses a two-phase search approaches. In the first phase, denoted as exploration, a sequence of solutions is generated by applying perturbations to the current best solution. In the second phase, denoted as intensification, the current best solution is refined using an embedded heuristic strategy that allows to generate quality neighbors. The main objective of 
the ILS is to obtain better results than the ones obtained using repeated random trials of that heuristic strategy. Interested readers are directed to Stützle (1999); Martin et al. (2003).

The pseudo code of the ILS proposed is illustrated in the Algorithm 3. In the line 4 of the algorithm it is established that while the solution is infeasible, for the EVRPB, typical and simple neighborhood structures will be applied to achieve feasibility. There are a large number of neighborhood structures developed by several authors (Taillard, 1993). However, all of them are basically generated by two types of moves, so-called SWAP and INSERT (Van Breedam, 1994). In lines 5 and 6 of the algorithm these two types of movements are considered. In line 5, of the Algorithm 3, the SWAP movement is applied as a disturbance strategy. The SWAP is swapping the customers at the ith position and the $j$ th position in the permutation, $i \neq j$.

In step three of the OSPB shown in the Algorithm 2, the INSERT movement was applied implicitly, seeking an infeasibility improvement in the current solution. Therefore, in line 6 of the Algorithm 3 the INSERT movement is also used.

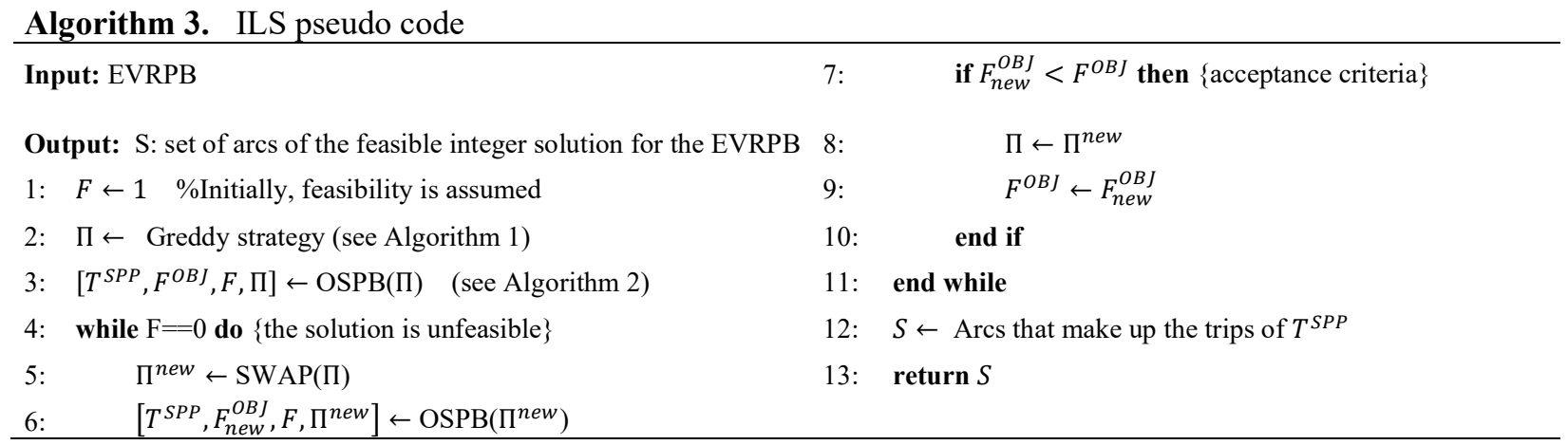

\section{Computational results}

The proposed model corresponds to a MILP formulation and was implemented in AMPL (Foureretal,1990) and solved with GUROBI 6.5 (called with the optimal gap option equal to $0 \%$ ), with a time limit of 14400 seconds, on a computer intel core i5-4210 2,4 Ghz, 4 GB of RAM. Two datasets of scenarios are used in order to show the operation and effectiveness of the proposed formulation. The first dataset, denoted as GJ dataset, was proposed by Goetschalckx and Jacobs-Blecha (1989) and contains 62 instances with a range between 20 and 150 customers. Details on how these scenarios were generated can be consulted in (Toth and Vigo, 2002). The second dataset, denoted as TV dataset, was proposed by Toth and Vigo (1997) and contains 33 instances between 21 and 100 customers. All the instances were kept with their original data, only the locations of the charging points of the set $K$ were added. The set of test instances for the EVRPB can be downloaded from http://academia.utp.edu.co/planeamiento/sistemas-de-prueba/.

Additionally, two types of results are presented. In order to show the efficiency of the proposed model, the first type of results corresponds to the direct solution of the MILP model using a commercial solver only. Because by making the battery life very long the proposed model can represent the standard VRPB, then a comparison of the our results versus the best-known solution (BKS) reported in the literature for the VRPB is presented too. The second type of results shows the performance of the ILS technique in those cases in which optimality was not reached in the direct EVRPB solution. In Table 1 and Table 2, the results for the VRPB using the GJ and TV datasets, respectively, are compared with those obtained by earlier methods, which are the stateof-the-art methods for VRPB instances from the literature (Toth and Vigo (1997); Mingozzi et al. (1999); Ropke and Pisinger (2006)). In Table 1, we give in columns 17: the problem name, the number of LCs, the number of BCs, the number of CPs, the capacity of the vehicle and the autonomy of the battery, respectively. The BKS obtained by earlier methods are presented 
in column 8. These methods correspond to the heuristic algorithm (HA) proposed by Ropke and Pisinger (2006), to the exact algorithm (TV) proposed by Toth and Vigo (1997) and to the exact algorithm (EHP) proposed by Mingozzi et al. (1999). The Euclidean distances were rounded to one decimal and the final result was rounded to an integer.

\section{Table 1}

Computational results for the VRPB cases from Goetschalckx and Jacobs-Blecha (1989)

\begin{tabular}{|c|c|c|c|c|c|c|c|c|c|c|c|c|c|c|c|c|c|}
\hline \multirow{3}{*}{$\frac{\mathrm{Id}}{\mathrm{A} 1}$} & \multirow{3}{*}{\multicolumn{2}{|c|}{$\begin{array}{ll}\mathrm{n} & \mathrm{m} \\
20 & 5\end{array}$}} & \multicolumn{4}{|c|}{ Instance } & \multirow[b]{2}{*}{ BKS } & \multicolumn{4}{|c|}{ MILP-VRPB } & \multicolumn{6}{|c|}{ MILP-EVRPB } \\
\hline & & & $\mathrm{k}$ & $K_{V}$ & Q & $E^{\max }$ & & Sol. & & ap & Time & Sol. & $K_{L B}$ & & p & $\tau_{1}$ & $t_{2}$ \\
\hline & & & 8 & 8 & 1,550 & 2,000 & $\mathrm{TV}^{\dagger}: 229,886$ & 229,886 & 5 & 0.00 & 1.85 & 238,725 & 5 & 3 & 0.00 & 1.61 & $\overline{45}$ \\
\hline 2 & 20 & 5 & 7 & 5 & 2,550 &, 000 & $\mathrm{TV}^{\dagger}: 180,119$ & 0,119 & 4 & 0.00 & 2.70 & 96,755 & 5 & & 0.00 & & 212 \\
\hline 3 & 20 & 5 & 3 & 4 & 050 & & & & 4 & 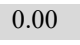 & & 88,439 & 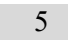 & & 0.00 & 3 & 19 \\
\hline 4 & 20 & 5 & 5 & 3 & 4,050 &, 000 & 96 & 5,796 & 3 & 0.00 & .24 & 61,438 & 3 & 1 & 0.00 & .05 & , \\
\hline 1 & 20 & 10 & 3 & 7 & 1,600 & 0,000 & $\mathrm{TV}^{\dagger}: 239,080$ & 9,080 & 6 & 00 & 16.35 & 249,239 & 7 & 2 & 0.00 & 0.06 & 350 \\
\hline 2 & 20 & 10 & 6 & 5 & 2,600 & 0,000 & & 8,048 & 5 & 0.00 & 2.14 & 10,792 & 5 & 3 & 0.00 & 04.25 & 506 \\
\hline 3 & 20 & 10 & 7 & 3 & 4,000 & & $\mathrm{TV}^{\dagger}:$ & & 3 & 0.00 & 5 & 69,634 & 5 & & 0.00 & 0.35 & \\
\hline 1 & 20 & 20 & 5 & 7 & 800 & & $\mathrm{TV}^{\dagger}: 249$ & 0,557 & 7 & 0.00 & 2.00 & 276 & 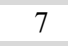 & & 0.00 & .61 & 42 \\
\hline 2 & 20 & 20 & 7 & 5 & 2,600 & & 20 & 5,020 & 5 & .00 & 4.82 & 450 & 5 & 4 & 0.00 & .02 & 699 \\
\hline ? & 20 & 20 & 7 & 5 & 4,150 & & & 9,346 & 4 & 0.00 & & 20,125 & 5 & 5 & 0.00 & & 487 \\
\hline 4 & 20 & 20 & 8 & 4 & 4,150 & & &, 366 & 4 & 0.00 & & & 4 & 3 & 0.00 & & 351 \\
\hline 1 & 30 & 8 & 9 & 12 & 700 & & & 30 & 6 & 0. & & & 5 & & 0.00 & & 1,250 \\
\hline 2 & 30 & 8 & 5 & 11 & 700 & & & & 6 & 0.6 & & & & & 0.00 & & 500 \\
\hline 3 & 30 & 8 & 6 & 7 & 2,750 & & TV & 9,479 & 4 & .00 & 7.04 & 32 & 7 & 4 & 0.00 & & 316 \\
\hline 4 & 30 & 8 & 7 & 5 & 4,075 & & & & 4 & 0.0 & & & 5 & 3 & 0.00 & & 61.05 \\
\hline 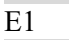 & 30 & 15 & 5 & 7 & 2,650 & & & & 4 & 0 & & & 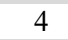 & 2 & 0.00 & & \\
\hline 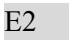 & 30 & 15 & 5 & I & & & & & 4 & 0 & & & 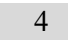 & & 0.00 & & \\
\hline 3 & 30 & 15 & 8 & 4 & 25 & & & & 3 & 0.0 & & & I & I & 0.00 & & \\
\hline 1 & 30 & 30 & 5 & 6 & 3,000 & & & & 6 & 0.00 & & & 6 & 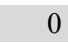 & 0.00 & & 00 \\
\hline & 30 & 30 & 7 & 7 & 3,000 & & TVt. 26 & & 7 & 0.0 & & & 7 & 4 & 0.00 & & 4,350 \\
\hline & 30 & 30 & 10 & 5 & & & TV & & 5 & & & & 5 & & 0.00 & & \\
\hline 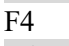 & 30 & 30 & 10 & 4 & & & & & 4 & 0.1 & & & $\sigma^{\circ}$ & & 0.00 & & \\
\hline 1 & 45 & & 7 & 10 & 00 & & GHP & & 6 & 0.0 & & & 6 & 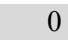 & 2.98 & & \\
\hline 0 & 45 & 12 & 3 & 6 & 00 & & & & 4 & 0.0 & & & 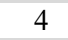 & 1 & 8.78 & & 000 \\
\hline & 45 & 12 & 5 & 5 & 5,300 & & TV & & 4 & & & & 4 & 1 & 11.68 & & \\
\hline $4^{\dagger}$ & 45 & 12 & 2 & 6 & & & & & 4 & & & & 6 & 0 & 8.87 & & \\
\hline 5 & 45 & 12 & 7 & 5 & & & & & 4 & 0.0 & & & 4 & 1 & 0.00 & & \\
\hline 6 & 45 & & 3 & 4 & 00 & & & & 4 & 0.00 & 5 & & 4 & 2 & 1.33 & & 400 \\
\hline & 45 & 23 & 4 & 6 & 4,000 & & & & 4 & & & & 5 & 0 & 0.00 & & 13,900 \\
\hline & 45 & 23 & 4 & 5 & & & & & 4 & & & & 5 & 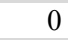 & 0.00 & & \\
\hline 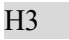 & 45 & 23 & 4 & 4 & & & & & 4 & 0.6 & & & 4 & 1 & 0. & & \\
\hline 4 & 45 & 23 & 6 & 5 & & & 1 & & 4 & 0.0 & & & 5 & 1 & 0.00 & 03 & 915.01 \\
\hline 5 & 45 & 23 & 5 & . & & & & & 4 & 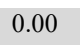 & & & 4 & 1 & 0.00 & .01 & 1,091 \\
\hline r & 45 & 23 & 5 & 5 & 7,100 & & $\mathrm{TV}^{\dagger}: 24$ & & 4 & & & & 5 & 1 & 0.00 & 00 & 1,760 \\
\hline & 45 & 45 & 5 & 10 & & & & & 10 & & & & 10 & 0 & 2.60 & & \\
\hline & 45 & 45 & 9 & 7 & & & 1.5 & & 7 & & & & 7 & J & 0.00 & & \\
\hline 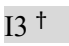 & 45 & 45 & 10 & 5 & & & & & 5 & & & & 5 & 2 & 0.00 & & 12,684 \\
\hline$\left[4^{\dagger}\right.$ & 45 & 45 & 8 & 6 & & & & & 6 & $0.00^{\dagger}$ & 137.89 & & 6 & 1 & 0.00 & & 4,710 \\
\hline I5t & 45 & 45 & 8 & 7 & 5,700 & & & & 7 & $0.00^{\dagger}$ & & & 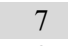 & 1 & 0.00 & .05 & 3,328 \\
\hline & 75 & 19 & 7 & 10 & & & & & 8 & & & & 8 & 0 & 9.43 & & \\
\hline & 75 & 19 & 8 & 8 & & & & & 8 & & & & 8 & 0 & 4.95 & $2,300.01$ & \\
\hline & 75 & 19 & 11 & 6 & 8,200 & & 9 & & 6 & $0.00^{\dagger}$ & & & 6 & 1 & 0.00 & 0.09 & \\
\hline & 75 & 19 & 6 & 7 & & & & & 7 & & & & 6 & 0 & 5.94 & & \\
\hline K1 & 75 & 38 & 12 & 10 & 4,100 & & & $4,071^{\dagger \dagger}$ & 9 & & & & ? & 3 & 15.40 & .21 & \\
\hline $\mathrm{K} 2^{\dagger}$ & 75 & 38 & 10 & 8 & 5,200 & 55,000 & HA: 362,130 & 362,130 & 7 & $0.00^{\dagger}$ & (1) & 381,504 & 0 & 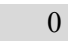 & 8.31 & $2,804.65$ & 14,400 \\
\hline $\mathrm{K} 3^{\dagger}$ & 75 & 38 & 14 & 9 & 5,200 & 48,000 & EHP: 365,694 & 365,694 & 7 & & 4,985 & 378,576 & 6 & 1 & 6.98 & $3,000.00$ & 14,400 \\
\hline$\underline{\mathrm{K}} 4^{\dagger}$ & 75 & 38 & 6 & 7 & 6,200 & 62,000 & HA: 348,950 & 348,950 & 6 & $0.00^{\dagger}$ & 6,530 & 378,049 & 6 & 3 & 10.30 & $1,200.54$ & 14,400 \\
\hline
\end{tabular}

BKS: best known solution values reported by first time.

MILP-VRPB: results for the MILP proposed model, considering an unlimited autonomy of the battery

MILP-EVRPB: results for the EVRPB using the MILP proposed model.

$K_{V}$ : number of available vehicles to use (given in advance)

$K_{L B}$ : number of vehicles performing routes conformed by linehaul and backhaul customers.

$K_{C P}$ : number of charge points visited by the electric vehicles.

Gap (\%): percentage gap is calculated as $(z-L B) / L B$.

$t_{1}$ : computing time until finding the first feasible integer solution. $t_{2}$ : overall computing time.

${ }^{\dagger}$ : optimality proven for the first time. ${ }^{\dagger+}$ : new BKS 
Table 2

Computational results for the VRPB cases from Toth and Vigo (1997)

\begin{tabular}{|c|c|c|c|c|c|c|c|c|c|c|c|c|c|c|c|c|c|c|}
\hline \multirow{3}{*}{$\begin{array}{r}\text { Id } \\
\text { FII } 2250 \mathrm{~A}\end{array}$} & \multicolumn{4}{|c|}{ Instance } & \multirow[b]{2}{*}{ Q } & \multirow[b]{2}{*}{$E^{\max }$} & & & \multicolumn{4}{|c|}{$\operatorname{MILP}-V R P B\left(E^{\max } \rightarrow \infty\right)$} & \multicolumn{6}{|c|}{ MILP-EVRPB } \\
\hline & $\mathrm{n}$ & $\mathrm{m}$ & $\mathrm{k}$ & $K_{V}$ & & & \multicolumn{2}{|c|}{$\mathrm{BKS}$} & Sol. & $K_{L B}$ & Gap & Time & Sol. & $K_{L B}$ & $K_{C P}$ & Gap & $t_{1}$ & $t_{2}$ \\
\hline & 11 & 10 & 2 & 3 & 6,000 & 120 & $\mathrm{TV}^{\dagger}:$ & 371 & 371 & 3 & 0.00 & 0.01 & 379 & 3 & 1 & 0.00 & 0.02 & 1 \\
\hline EIL2266A & 14 & 7 & 2 & 3 & 6,000 & 100 & $\mathrm{TV}^{\dagger}:$ & 366 & 366 & 3 & 0.00 & 0.01 & 430 & 3 & 2 & 0.00 & 0.04 & 15 \\
\hline EIL2280A & 17 & 4 & 4 & 3 & 6,000 & 100 & $\mathrm{TV}^{\dagger}:$ & 375 & 375 & 3 & 0.00 & 0.01 & 394 & 2 & 2 & 0.00 & 5.04 & 30 \\
\hline EIL2350A & 11 & 11 & 5 & 2 & 4,500 & 250 & $\mathrm{TV}^{\dagger}$ : & 682 & 682 & 2 & 0.00 & 0.01 & 706 & 2 & 2 & 0.00 & 0.01 & 2 \\
\hline EIL2366A & 15 & 7 & 4 & 2 & 4,500 & 250 & $\mathrm{TV}^{\dagger}:$ & 649 & 649 & 2 & 0.00 & 0.01 & 667 & 2 & 1 & 0.00 & 0.03 & 5 \\
\hline EIL2380A & 18 & 4 & 3 & 2 & 4,500 & 200 & $\mathrm{TV}^{\dagger}$ : & 623 & 623 & 2 & 0.00 & 0.01 & 671 & 2 & 1 & 0.00 & 0.01 & 2 \\
\hline EIL3050A & 15 & 14 & 4 & 2 & 4,500 & 250 & $\mathrm{TV}^{\dagger}:$ & 501 & 501 & 2 & 0.00 & 0.01 & 506 & 2 & 1 & 0.00 & 4.91 & 50 \\
\hline EIL3066A & 20 & 9 & 7 & 3 & 4,500 & 200 & $\mathrm{TV}^{\dagger}:$ & 537 & 537 & 3 & 0.00 & 0.30 & 537 & 3 & 0 & 0.00 & 1.87 & 25 \\
\hline EIL3080A & 24 & 5 & 4 & 3 & 4,500 & 180 & $\mathrm{TV}^{\dagger}:$ & 514 & 514 & 3 & 0.00 & 0.30 & 518 & 3 & 1 & 0.00 & 1.82 & 20 \\
\hline EIL3350A & 16 & 16 & 5 & 3 & 8,000 & 300 & $\mathrm{TV}^{\dagger}:$ & 738 & 738 & 2 & 0.00 & 0.14 & 742 & 2 & 1 & 0.00 & 0.01 & 1 \\
\hline EIL3366A & 22 & 10 & 3 & 3 & 8,000 & 250 & $\mathrm{TV}^{\dagger}:$ & 750 & 750 & 2 & 0.00 & 0.38 & 758 & 2 & 2 & 0.00 & 1.02 & 2 \\
\hline EIL3380A & 26 & 6 & 4 & 3 & 8,000 & 300 & $\mathrm{TV}^{\dagger}:$ & 736 & 736 & 3 & 0.00 & 7.31 & 859 & 3 & 0 & 0.00 & 0.03 & 1 \\
\hline EIL5150A & 25 & 25 & 5 & 3 & 160 & 250 & $\mathrm{TV}^{\dagger}:$ & 559 & 559 & 3 & 0.00 & 1.80 & 559 & 3 & 0 & 0.00 & 2.01 & 5 \\
\hline EIL5166A & 34 & 16 & 7 & 4 & 160 & 150 & $\mathrm{TV}^{\dagger}:$ & 548 & 548 & 4 & 0.00 & 2.01 & 553 & 4 & 1 & 0.00 & 10.56 & 58 \\
\hline EIL5180A & 40 & 10 & 6 & 4 & 160 & 200 & $\mathrm{TV}^{\dagger}:$ & 565 & 565 & 3 & 0.00 & 26.11 & 569 & 3 & 1 & 0.00 & 2.51 & 3,250 \\
\hline EILA7650A & 37 & 38 & 4 & 6 & 140 & 350 & $\mathrm{TV}^{\dagger}:$ & 739 & 739 & 6 & 0.00 & 64.21 & 760 & 6 & 0 & 0.00 & 18.52 & 747 \\
\hline EILA7666A & 50 & 25 & 5 & 7 & 140 & 350 & $\mathrm{TV}^{\dagger}:$ & 768 & 768 & 6 & 0.00 & 743.00 & 768 & 6 & 0 & 0.00 & 2.09 & 10,820 \\
\hline EILA7680A & 60 & 15 & 4 & 8 & 140 & 200 & TV: & 781 & 781 & 5 & 0.99 & 14,400 & 802 & 5 & 1 & 5.80 & 328.21 & 14,400 \\
\hline EILB7650A & 37 & 38 & 5 & 8 & 100 & 120 & $\mathrm{TV}^{\dagger}:$ & 801 & 801 & 7 & 0.00 & 40.96 & 812 & 7 & 2 & 0.00 & 2.08 & 12,850 \\
\hline EILB7666A & 50 & 25 & 5 & 10 & 100 & 450 & $\mathrm{TV}^{\dagger}:$ & 873 & 873 & 8 & 0.94 & 14,400 & 873 & 8 & 0 & 2.50 & 3.01 & 14,400 \\
\hline EILB7680A & 60 & 15 & 8 & 12 & 100 & 450 & $\mathrm{TV}^{\dagger}:$ & 919 & 933 & 6 & 3.92 & 14,400 & 934 & 5 & 1 & 5.40 & 2.10 & 14,400 \\
\hline EILC7650A & 37 & 38 & 6 & 5 & 180 & 150 & $\mathrm{TV}^{\dagger}:$ & 713 & 713 & 5 & 0.00 & 8.64 & 720 & 5 & 0 & 0.00 & 22.00 & 13,521 \\
\hline EILC7666A & 50 & 25 & 2 & 6 & 180 & 120 & $\mathrm{EHP}^{\dagger}$ & 734 & 734 & 6 & 0.00 & 185.00 & 763 & 6 & 1 & 0.00 & 10.01 & 9,800 \\
\hline EILC7680A & 60 & 15 & 5 & 7 & 180 & 350 & TV: & 733 & 733 & 5 & 1.50 & 14,400 & 747 & 4 & 1 & 4.62 & 2.01 & 14,400 \\
\hline EILD7650A & 37 & 38 & 6 & 4 & 220 & 150 & $\mathrm{TV}^{\dagger}:$ & 690 & 690 & 4 & 0.00 & 6.03 & 703 & 4 & 3 & 0.00 & 3.03 & 653 \\
\hline EILD7666A $^{\dagger}$ & 50 & 25 & 10 & 5 & 220 & 150 & TV: & 715 & 715 & 5 & $0.00^{\dagger}$ & 32.54 & 721 & 5 & 1 & 0.00 & 12.09 & 4,015 \\
\hline EILD7680A ${ }^{\dagger}$ & 60 & 15 & 5 & 6 & 210 & 170 & EHP: & 694 & 694 & 4 & $0.00^{\dagger}$ & 845.00 & 701 & 5 & 0 & 0.00 & 4.54 & 12,800 \\
\hline EILA $10150 \mathrm{~A}^{\dagger}$ & 50 & 50 & 6 & 4 & 200 & 450 & HA: & 831 & 831 & 4 & $0.00^{\dagger}$ & 938.00 & 844 & 5 & 1 & 3.18 & 3.63 & 14,400 \\
\hline EILA10166A & 67 & 33 & 5 & 6 & 200 & 180 & $\mathrm{TV}^{\dagger}:$ & 846 & 846 & 6 & 0.00 & 6.00 & 851 & 6 & 1 & 0.00 & 2.32 & 1,750 \\
\hline EILA10180A & 80 & 20 & 7 & 7 & 200 & 100 & HA: & 857 & 859 & 6 & 0.82 & 14,400 & 938 & 5 & 4 & 9.41 & 6.38 & 14,400 \\
\hline EILB10150A ${ }^{\dagger}$ & 50 & 50 & 8 & 7 & 112 & 240 & HA: & 925 & $923^{\dagger+}$ & 7 & $0.00^{\dagger}$ & 792 & 983 & 7 & 0 & 10.60 & 3,900 & 14,400 \\
\hline EILB10166A & 67 & 33 & 3 & 10 & 112 & 200 & HA: & 989 & $971^{\dagger \dagger}$ & 8 & 2.99 & 14,400 & 984 & 8 & 0 & 5.53 & 10.91 & 14,400 \\
\hline EILB10180A & 80 & 20 & 7 & 11 & 112 & 850 & HA: & 1,008 & 1013 & 9 & 1.46 & 14,400 & 1,023 & 8 & 0 & 5.20 & 28.00 & 14,400 \\
\hline
\end{tabular}

The nomenclature of this table is the same as that presented in Table 1 .

In columns 9-12 the characteristics of the solution for the standard VRPB are presented. The solution obtained by solving the MILP model are presented in column 9. Column 10 shows the number of vehicles $K_{L B}$ performing routes conformed by linehaul and backhaul customers. Thus, $K_{V}-K_{L B}$ is the number of vehicles performing routes conformed by linehaul customers exclusively (see Figure 6). Columns 11 and 12 show the percentage gap and computing time (expressed in CPU seconds), respectively. In columns 13-18 the results obtained by solving the MILP model for the EVRPB are presented. The number of recharging points $\left(K_{C P}\right)$, used to recharge the EV batteries, are presented in column 15 . Columns 17 and 18 show, respectively, the time it takes for the solver to find the first feasible integer solution and the total computation time until reaching the reported solution.

Regarding the standard VRPB, when $E^{\mathrm{max}}$ is considered large enough, the optimality for the GJ dataset was proved for the first time for 8 instances. One new best-known solutions was found for the instance k1. Optimality was achieved in 42 of the 47 scenarios (see Table 1). For the TV dataset the optimality of 4 TV instances is proven for first time. Two new best-known solutions were found considering both heuristic methods and exact methods. Optimality was achieved in 27 of the 33 cases (see Table 2). As can be seen from the computational results, the proposed model produces high quality results, obtaining equal or better upper bounds in all instances, and the final lower bounds prove stronger than those obtained by earlier methods.

Regarding the EVRPB, the optimality for the GJ dataset was achieved in 34 of the 47 intances and for the TV dataset, the optimality was achieved in 24 of the 33 intances. It is interesting to note that in some instances the number $K_{C P}$ is 0 , which implies that the EVs did not have the need to visit CPs. However, in some of these cases, such as the instance H1, the optimal solution found for the EVRPB differs from that found for the VRPB. This happens because, in the case of the EVRPB, the autonomy of the battery forces the model to generate a different solution, even using a different number of vehicles $K_{L B}$, with an objective function of greater value.

Note that among the instances for which it was not possible to achieve optimality, the instances G1, G2, G3, G4, G6, I1, J1, J2, J4, K1, K2, K3, K4, EILA7680A and EILB10150A present the longest times $t_{1}$. Therefore this set of instances is quite interesting to be addressed with the initialization methodology based on ILS. Table 3 shows 
a comparison of the computational results obtained with the proposed mathematical model, the ILS technique and the model with initialization methodology. It can be seen that the proposed ILS technique is quite efficient to find a feasible integer solution in a short time, as shown in columns 6 and 7 of the table. However, in 14400 seconds the ILS technique, by itself, fails to achieve the results obtained with the exact model, as observed when comparing columns 2 and 5 of the table. Finally, when the first feasible integer solution (FFIS) found efficiently by the ILS method is used, the results obtained in 14400 seconds surpass all the previous ones, as can be seen in columns 8 and 9 of the table. Note that for the G6 instance the methodology reached optimality. The detailed routes of the optimal solution of this instance are shown in Figure 6. In Table 4, the detailed routes of the solutions of the aforementioned instances for the EVRPB are shown.

Table 3

Comparison of the computational results obtained with the proposed mathematical model, the ILS technique and the model with initialization methodology

\begin{tabular}{|c|c|c|c|c|c|c|c|c|c|}
\hline \multirow[b]{2}{*}{ Instance } & \multicolumn{3}{|c|}{ MILP-EVRPB from Tables 1 and 2} & \multirow{2}{*}{$\begin{array}{r}\text { ILS } \\
\text { Sol }^{a} \text {. }\end{array}$} & \multicolumn{5}{|c|}{ MILP-EVRPB with initialization methodology } \\
\hline & $\mathrm{Sol}^{a}$. & Gap $(\%)$ & $t_{1}$ & & FFIS & $t_{3}$ & Sol $^{b}$. & Gap $(\%)$ & $t_{2}$ \\
\hline G1 & 308,779 & 2.98 & $1,628.21$ & 356,854 & 401,562 & 0.02 & 307,965 & 1.63 & 14,400 \\
\hline G2 & 265,498 & 8.78 & $2,304.00$ & 368,951 & 427,632 & 0.01 & 262,126 & 7.20 & 14,400 \\
\hline G3 & 252,495 & 11.68 & $2,498.62$ & 321,458 & 456,554 & 0.01 & 248,214 & 6.67 & 14,400 \\
\hline G4 & 262,976 & 8.87 & $2,265.32$ & 314,284 & 434,842 & 0.08 & 261,306 & 8.12 & 14,400 \\
\hline G6 & 225,829 & 1.33 & $1,278.00$ & 327,773 & 417,266 & 0.95 & 224,966 & 0.00 & 8,997 \\
\hline I1 & 351,553 & 2.60 & $2,332.21$ & 424,681 & 586,442 & 3.53 & 350,814 & 1.10 & 14,400 \\
\hline $\mathrm{J} 1$ & 349,639 & 9.43 & $1,232.00$ & 382,459 & 526,168 & 1.04 & 344,192 & 5.80 & 14,400 \\
\hline $\mathrm{J} 2$ & 315,408 & 4.95 & $2,300.01$ & 428,624 & 501,451 & 1.23 & 313,486 & 3.82 & 14,400 \\
\hline $\mathrm{J} 4$ & 299,376 & 5.94 & $1,260.32$ & 379,915 & 482,714 & 0.09 & 297,766 & 2.43 & 14,400 \\
\hline $\mathrm{K} 1$ & 452,086 & 15.40 & $1,800.21$ & 488,922 & 612,456 & 14.02 & 421,546 & 7.60 & 14,400 \\
\hline $\mathrm{K} 2$ & 381,504 & 8.31 & $2,804.65$ & 428,561 & 594,821 & 8.65 & 378,744 & 7.53 & 14,400 \\
\hline K3 & 378,576 & 6.98 & $3,000.00$ & 394,522 & 602,451 & 10.02 & 366,134 & 3.91 & 14,400 \\
\hline K4 & 378,049 & 10.30 & $1,200.54$ & 398,205 & 598,428 & 16.32 & 355,109 & 3.30 & 14,400 \\
\hline EILA7680A & 802 & 5.80 & 328.21 & 1,088 & 1,355 & 3.04 & 799 & 4.89 & 14,400 \\
\hline EILB10150A & 983 & 10.60 & $3,900.21$ & 1,224 & 1,550 & 2.04 & 934 & 4.76 & 14,400 \\
\hline
\end{tabular}

$\mathrm{Sol}^{a}$ : solution obtained in 14,400 seconds. Sol ${ }^{b}$ : solution obtained in $t_{2}$ seconds (overall computing time).

$t_{1}$ : computing time until finding the first feasible integer solution.

FFIS: first feasible integer solution found by the ILS method in $t_{3}$ seconds.

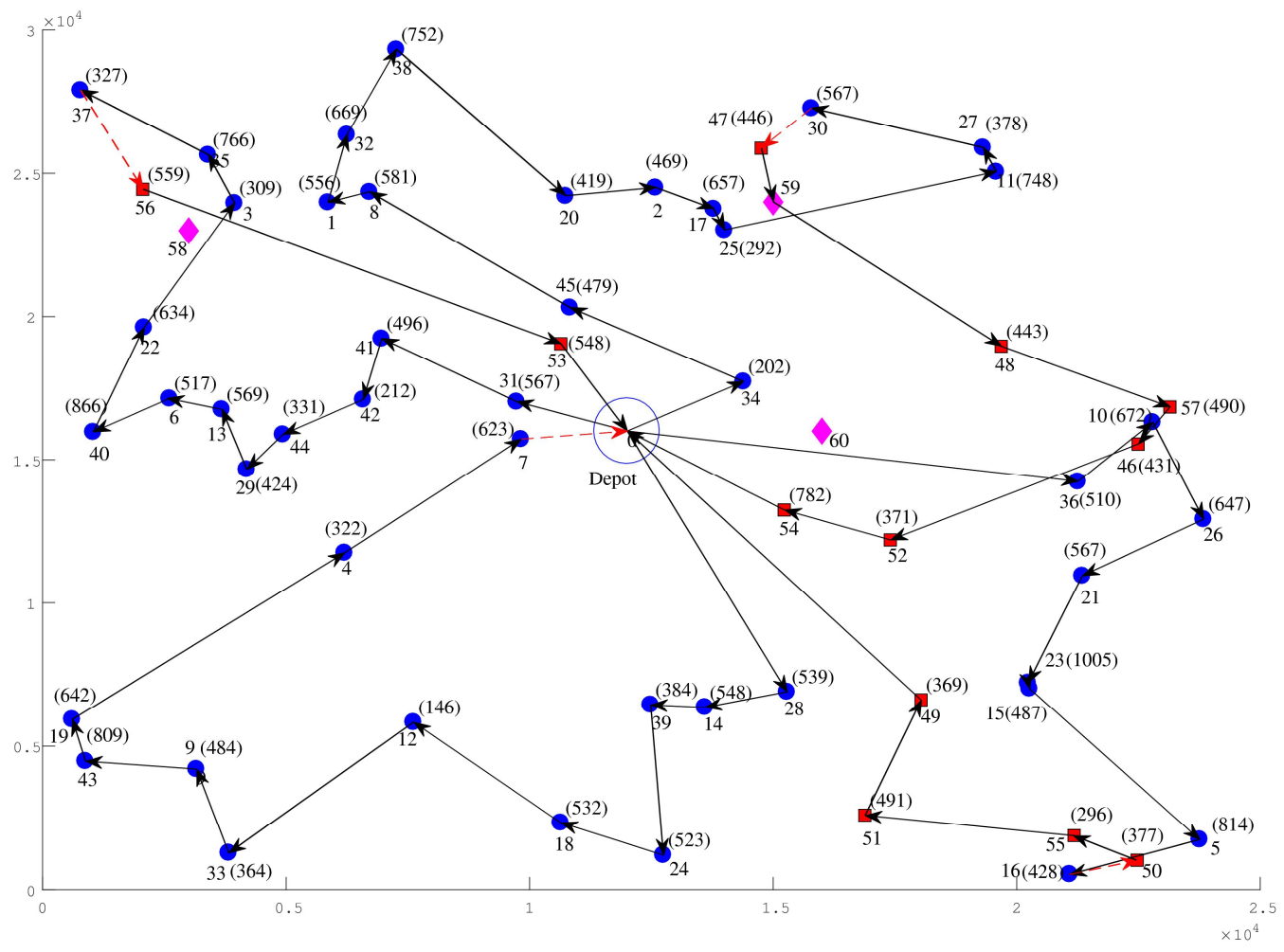

Fig. 6. Optimal solution of the instance G6 found using the MILP-EVRPB with initialization methodology 


\section{Conclusions and future works}

In this paper, we have presented a new general-purpose model for modeling the EVRPB by mixed integer programming. The results show that the proposed commodity flow model produces good quality solutions and allows to resolve efficiently instances proposed in the literature with up to 150 customers. The EVRPB is a problem of current interest that occurs in transport companies with fleets of electric vehicles that collect and deliver goods to customers. In relation to the standard VRPB it is appreciated that the EVRPB is a problem of greater complexity and much more restricted when it comes to finding a feasible integer solution. Thus, an iterated local search algorithm is presented for to obtain quickly and effectively a feasible integer solution of the EVRPB. When this solution is used as the upper limit in the solution of larger and more complex instances, the results obtained are much better than those obtained independently by the ILS technique or by the MIP model. We believe that from the point of view of the exact model, the results could further improve with the inclusion of bounding techniques or setpartitioning models, which could significantly increase the size and number of the instances solvable by exact techniques. From the point of view of heuristic techniques, the optimal splitting procedure of a permutation that we propose can be widely adapted and improved to much more elaborated heuristic techniques that allow to reach better solutions in instances of greater size.

\section{Table 4}

Detailed routes for the EVRPB

\begin{tabular}{|c|c|}
\hline Instance & Sequence $($ Blue points $=$ LCs; red points $=$ BCs; magenta points $=$ CPs $)$ \\
\hline G1: & $\begin{array}{l}r_{1}=4244223353756 ; r_{2}=391824165505551 ; r_{3}=3436212610465748 ; r_{4}=314122045 ; r_{5}=2517112730 \\
47 ; r_{6}=14282315495254 ; r_{7}=123394319 ; r_{8}=7 ; r_{9}=42940613 ; r_{10}=18323853\end{array}$ \\
\hline G2: & 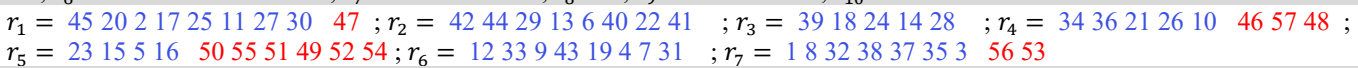 \\
\hline G3: & 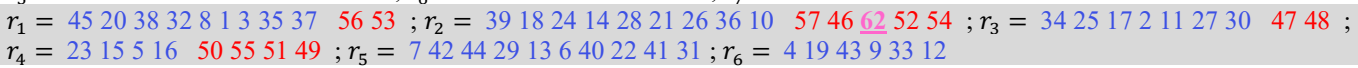 \\
\hline G4: & $\begin{array}{l}r_{1}=45203832813353756 ; r_{2}=391824142854 ; r_{3}=3425172112730 \quad 47 ; r_{4}=31424429136402241 \\
53 ; r_{5}=23155165055514952 ; r_{6}=21263610 \quad 465748 ; r_{7}=74194393312\end{array}$ \\
\hline G6: & $\begin{array}{l}r_{1}=36102621231551650555149 ; r_{2}=34458132382021725112730 \quad 47 \underline{59} 4857465254 ; r_{3}=314142 \\
4429136402233537 \quad 5653 ; r_{4}=281439241812339431947\end{array}$ \\
\hline I1: & $\begin{array}{l}r_{1}=39228323858895785619256 ; r_{2}=36454131 \quad 74477990 ; r_{3}=34537353467581656451 ; r_{4}=33 \\
63 ; r_{5}=3029241316782886776 ; r_{6}=19220212315877153506669 ; r_{7}=10402617256055844959 ; \\
r_{8}=94383736877 ; r_{9}=64442411278670 \underline{93} 6272 ; r_{10}=1281418125452784880\end{array}$ \\
\hline J1: & $\begin{array}{l}r_{1}=7439326684914639383 ; r_{2}=557572563836289 ; r_{3}=37126954 ; r_{4}=34607047432517164879 \\
88 ; r_{5}=3346527364282290 ; r_{6}=3130624018673537 ; r_{7}=10231553451942692 ; r_{8}=958657168 \\
11278178808776 ; r_{9}=561459514421 \quad 779491 ; r_{10}=12457264150202913 \quad 9685868284\end{array}$ \\
\hline $\mathbf{J} 2$ & $\begin{array}{l}r_{1}=74346070474325171648 \quad 7988 ; r_{2}=557572563836289 ; r_{3}=37546939326684914639383 ; r_{4}= \\
33958514465716811278178808776 ; r_{5}=1222642194553152310 ; r_{6}=731304033567186221 \quad 7794 \\
91 ; r_{7}=56145946527364289092 ; r_{8}=1245726415020132985868284\end{array}$ \\
\hline J4 & $\begin{array}{l}r_{1}=725638363356718406221779491 ; r_{2}=60161725484347706314498326683937988 ; r_{3}=5574 \\
3475289 ; r_{4}=3754693926415020132985868284 ; r_{5}=1012457231553451942622 \quad 9092 ; r_{6}=731 \\
305958514465716811278178808776 ; r_{7}=5614946527364283312\end{array}$ \\
\hline K1 & $\begin{array}{l}r_{1}=705345711488101 ; r_{2}=60132919646220428393102 \underline{115} 999779111 ; r_{3}=58696327374348492 \\
90108 ; r_{4}=4923164868571 \quad 11077 ; r_{5}=44557313843 \quad 10682868178 ; r_{6}=3964737 ; r_{7}=35675030 \\
72561026105 ; r_{8}=2425176646218511280104107 ; r_{9}=1554285918515241 \quad 1039411391109 ; r_{10}=12 \\
65759403310095969889 ; r_{11}=11273661522328 \quad 7687 ; r_{12}=34\end{array}$ \\
\hline K2 & $\begin{array}{l}r_{1}=6013291964622042339409695100839310211599977988101 ; r_{2}=557313843102672563077110 \\
94 ; r_{3}=4948166815741 \quad 103113911099889 ; r_{4}=395869632737434849290108 ; r_{5}=242517664621 \\
851128610682817880104107 ; r_{6}=1554285918515223 ; r_{7}=1265751471455370111 ; r_{8}=11273661 \\
522326374787687 ; r_{9}=4335446750 \quad 105\end{array}$ \\
\hline K3 & 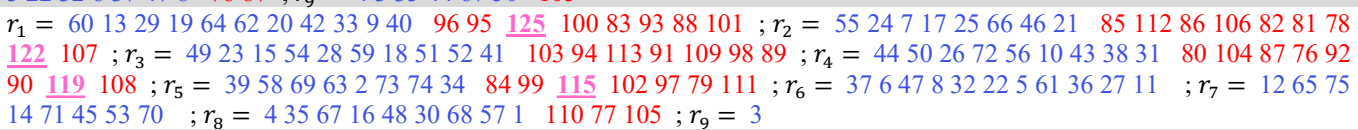 \\
\hline K4 & $\begin{array}{l}r_{1}=705345711488101799799102938310095969889 ; r_{2}=583764769632737434849290108 ; r_{3}=39 \\
55738311725664621851128610682817880104107 ; r_{4}=231554285918515241685711109410311391 \\
109 ; r_{5}=126575940334220626419291360111 ; r_{6}=435674424271136615223287687 ; r_{7}=34948 \\
165026431056723077105\end{array}$ \\
\hline EILB10150A: & $\begin{array}{l}r_{1}=48305047315810099969897 ; r_{2}=4542392310632956655819476 ; r_{3}=39215401741 \quad 896762 \\
908456 ; r_{4}=28132034123861878678527763 ; r_{5}=2711372129822 \quad 71517079 ; r_{6}=16424258268 \\
7354749159 ; r_{7}=1435126518333683606585758864 ; r_{8}=74449194346 \\
72576993928053\end{array}$ \\
\hline EILA7680A: & $\begin{array}{l}r_{2}=55250234956293057727464636169 \quad r_{1}=377164411462242 ; r_{2}=2854 \quad ; r_{3}=2759135333452 \\
18 \quad ; r_{4}=2147582531537362 ; r_{5}=143362683210 \quad ; r_{6}=64394812 \quad 67 ; r_{7}=54113511945402015 \\
71657068 ; r_{8}=424393817606675\end{array}$ \\
\hline
\end{tabular}




\section{References}

Alinaghian, M., Kalantari, M. R., Bozorgi-Amiri, A., \& Raad, N. G. (2016). A novel mathematical model for cross dock open-close vehicle routing problem with splitting. Int. J. Math. Sci. Comput.(IJMSC), 2(3), 21-31.

Arias, A., Granada, M., \& Castro, C. A. (2017). Optimal probabilistic charging of electric vehicles in distribution systems. IET Electrical Systems in Transportation, 7(3), 246-251.

Bodin, L. (1983). Routing and scheduling of vehicles and crews, the state of the art. Comput. Oper. Res., 10(2), 63-211.

Cattaruzza, D., Absi, N., Feillet, D., \& Vidal, T. (2014). A memetic algorithm for the multi trip vehicle routing problem. European Journal of Operational Research, 236(3), 833-848.

Chávez, J., Escobar, J., \& Echeverri, M. (2016). A multi-objective Pareto ant colony algorithm for the Multi-Depot Vehicle Routing problem with Backhauls. International Journal of Industrial Engineering Computations, 7(1), 35-48.

Chávez, J., Escobar, J., Echeverri, M., \& Meneses, C. (2018). A heuristic algorithm based on tabu search for vehicle routing problems with backhauls. Decision Science Letters, 7(2), 171-180.

Cormen, T. H., Leiserson, C. E., Rivest, R. L., \& Stein, C. (2001). Introduction to algorithms second edition. The Knuth-Morris-Pratt Algorithm, year.

Dharmakeerthi, C. H., Mithulananthan, N., \& Saha, T. K. (2012, July). Modeling and planning of EV fast charging station in power grid. In 2012 IEEE Power and Energy Society General Meeting (pp. 18). IEEE.

Electrification Coalition (2012) EV case study, the electric drive bellwether? - FedEx express on lessons learned - From global EV deployments. Tech. rep., Washington DC.

Fisher, M. L., Jaikumar, R., \& Van Wassenhove, L. N. (1986). A multiplier adjustment method for the generalized assignment problem. Management Science, 32(9), 1095-1103.

Fourer, R., Gay, D. M., \& Kernighan, B. W. (1990). A modeling language for mathematical programming. Management Science, 36(5), 519-554.

Ge, S., Feng, L., \& Liu, H. (2011, September). The planning of electric vehicle charging station based on grid partition method. In 2011 International Conference on Electrical and Control Engineering (pp. 2726-2730). IEEE.

Goeke, D., \& Schneider, M. (2015). Routing a mixed fleet of electric and conventional vehicles. European Journal of Operational Research, 245(1), 81-99.

Goetschalckx, M., \& Jacobs-Blecha, C. (1989). The vehicle routing problem with backhauls. European Journal of Operational Research, 42(1), 39-51.

Irnich, S., Schneider, M., \& Vigo, D. (2014). Chapter 9: Four Variants of the Vehicle Routing Problem. In Vehicle Routing: Problems, Methods, and Applications, Second Edition (pp. 241-271). Society for Industrial and Applied Mathematics.

Koç, Ç., \& Laporte, G. (2018). Vehicle routing with backhauls: Review and research perspectives. Computers \& Operations Research, 91, 79-91.

Letchford, A. N., Lysgaard, J., \& Eglese, R. W. (2007). A branch-and-cut algorithm for the capacitated open vehicle routing problem. Journal of the Operational Research Society, 58(12), 1642-1651.

Li, F., Golden, B., \& Wasil, E. (2007). The open vehicle routing problem: Algorithms, large-scale test problems, and computational results. Computers \& Operations Research, 34(10), 2918-2930.

Liu, S., Lei, L., \& Park, S. (2008). On the multi-product packing-delivery problem with a fixed route. Transportation Research Part E: Logistics and Transportation Review, 44(3), 350-360.

Liu, Z., Wen, F., \& Ledwich, G. (2012). Optimal planning of electric-vehicle charging stations in distribution systems. IEEE Transactions on Power Delivery, 28(1), 102-110.

Lourenco, H. R., Martin, O. C., \& Stuetzle, T. (2002). Handbook of Metaheuristics, chapter Iterated local search.

Mingozzi, A., Giorgi, S., \& Baldacci, R. (1999). An exact method for the vehicle routing problem with backhauls. Transportation Science, 33(3), 315-329. 
Ochi, L. S., Vianna, D. S., Drummond, L. M., \& Victor, A. (1998). A parallel evolutionary algorithm for the vehicle routing problem with heterogeneous fleet. Future Generation Computer Systems, 14(5-6), 285-292.

Parragh, S. N., Doerner, K. F., \& Hartl, R. F. (2008). A survey on pickup and delivery problems. Journal für Betriebswirtschaft, 58(1), 21-51.

Paz, J., Granada-Echeverri, M., \& Escobar, J. (2018). The multi-depot electric vehicle location routing problem with time windows. International Journal of Industrial Engineering Computations, 9(1), 123-136.

Pessoa, A., De Aragão, M. P., \& Uchoa, E. (2008). Robust branch-cut-and-price algorithms for vehicle routing problems. In The vehicle routing problem: Latest advances and new challenges (pp. 297-325). Springer, Boston, MA.

Pfriem, M., \& Gauterin, F. (2013, November). Less range as a possible solution for the market success of electric vehicles in commercial fleets. In 2013 World Electric Vehicle Symposium and Exhibition (EVS27) (pp. 1-8). IEEE.

Prins, C. (2004). A simple and effective evolutionary algorithm for the vehicle routing problem. Computers \& Operations Research, 31(12), 1985-2002.

Ropke, S., \& Pisinger, D. (2006). A unified heuristic for a large class of vehicle routing problems with backhauls. European Journal of Operational Research, 171(3), 750-775.

Salari, M., Toth, P., \& Tramontani, A. (2010). An ILP improvement procedure for the open vehicle routing problem. Computers \& Operations Research, 37(12), 2106-2120.

Schrage, L. (1981). Formulation and structure of more complex/realistic routing and scheduling problems. Networks, 11(2), 229-232.

Stützle, T. (1999). Local search algorithms for combinatorial problems-analysis, algorithms and new applications. DISKI-Dissertationen zur Künstliken Intelligenz. In x, Sankt Augustin, Germany.

Taillard, É. (1993). Parallel iterative search methods for vehicle routing problems. Networks, 23(8), 661673.

Toro, E. M., Franco, J. F., Echeverri, M. G., \& Guimarães, F. G. (2017a). A multi-objective model for the green capacitated location-routing problem considering environmental impact. Computers \& Industrial Engineering, 110, 114-125.

Toro, E., Franco, J., Echeverri, M., Guimarães, F., \& Rendón, R. (2017b). Green open location-routing problem considering economic and environmental costs. International Journal of Industrial Engineering Computations, 8(2), 203-216.

Toth, P., \& Vigo, D. (1997). An exact algorithm for the vehicle routing problem with backhauls. Transportation science, 31(4), 372-385.

Toth, P., \& Vigo, D. (Eds.). (2002). The vehicle routing problem. Society for Industrial and Applied Mathematics.

Toth, P., \& Vigo, D. (Eds.). (2014). Vehicle routing: problems, methods, and applications. Society for Industrial and Applied Mathematics.

Van Breedam, A. (1994). An Analysis of the Behavior of Heuristics for the Vehicle Routing Problem for a Selectrion of Problems with Vehicle-related, Customer-related, and Time-related Constraints. RUCA.

Vidal, T., Crainic, T. G., Gendreau, M., Lahrichi, N., \& Rei, W. (2012). A hybrid genetic algorithm for multidepot and periodic vehicle routing problems. Operations Research, 60(3), 611-624.

Wade, A., \& Salhi, S. (2003). An ant system algorithm for the mixed vehicle routing problem with backhauls. In Metaheuristics: computer decision-making (pp. 699-719). Springer, Boston, MA.

Wang, G., Xu, Z., Wen, F., \& Wong, K. P. (2013). Traffic-constrained multiobjective planning of electric-vehicle charging stations. IEEE Transactions on Power Delivery, 28(4), 2363-2372.

Yang, J., \& Sun, H. (2015). Battery swap station location-routing problem with capacitated electric vehicles. Computers \& Operations Research, 55, 217-232. 
(C) 2019 by the authors; licensee Growing Science, Canada. This is an open access article distributed under the terms and conditions of the Creative Commons Attribution (CCBY) license (http://creativecommons.org/licenses/by/4.0/). 